\title{
SOME LOGICAL INVARIANTS OF ALGEBRAS AND LOGICAL RELATIONS BETWEEN ALGEBRAS
}

\author{
B. PLOTKIN AND G. ZHITOMIRSKI
}

Dedicated to the centenary of D. K. Faddeev's birth

\begin{abstract}
Let $\Theta$ be an arbitrary variety of algebras and $H$ an algebra in $\Theta$. Along with algebraic geometry in $\Theta$ over the distinguished algebra $H$, a logical geometry in $\Theta$ over $H$ is considered. This insight leads to a system of notions and stimulates a number of new problems. Some logical invariants of algebras $H \in \Theta$ are introduced and logical relations between different $H_{1}$ and $H_{2}$ in $\Theta$ are analyzed. The paper contains a brief review of ideas of logical geometry $(\S 1)$, the necessary material from algebraic logic $(\S 2)$, and a deeper introduction to the subject (§3). Also, a list of problems is given.
\end{abstract}

0.1. Introduction. The paper consists of three sections. A reader wishing to get a feeling of the subject and to understand the logic of the main ideas can confine himself to $\S 1$. A more advanced look at the topic of the paper is presented in $\S \S 2$ and 3 .

In $\S 1$ we give a list of the main notions, formulate some results, and specify problems. Not all the notions used in $\S 1$ are well formalized and commonly known. In particular, we operate with algebraic logic, referring to $\S 2$ for precise definitions. However, $\S 1$ is self-contained from the viewpoint of ideas of universal algebraic geometry and logical geometry.

Old and new notions from algebraic logic are collected in $\S 2$. Here we define the Halmos categories and multisorted Halmos algebras related to a variety $\Theta$ of algebras.

$\S 3$ is a continuation of $\S 1$. Here we give necessary proofs and discuss problems. The main problem we are interested in is what are the algebras with the same geometrical logic.

The theory described in the paper has deep ties with model theory, and some problems are of a model-theoretic nature.

We emphasize once again that $\S 1$ gives a complete insight on the subject, while $\S 2$ and $\S 3$ describe and decode the material of $\S 1$.

\section{§1. Preliminaries. General view}

1.1. Main idea. We fix an arbitrary variety $\Theta$ of algebras. Throughout the paper we consider algebras $H$ in $\Theta$. To each algebra $H \in \Theta$ one can attach an algebraic geometry (AG) in $\Theta$ over $H$ and a logical geometry (LG) in $\Theta$ over $H$.

In algebraic geometry we consider algebraic sets over $H$, while in logical geometry we consider logical (elementary) sets over $H$. These latter sets are related to the elementary logic, i.e., to the first order logic (FOL).

Consideration of these sets gives grounds to geometries in an arbitrary variety of algebras. We distinguish algebraic and logical geometries in $\Theta$. However, there is very

2000 Mathematics Subject Classification. Primary 03G25.

Key words and phrases. Variety of algebras, algebraic geometry, logical geometry. 
little trace of the usual geometry in geometries of such a kind. It should be remembered that we consider an arbitrary variety $\Theta$. Only some "good" varieties $\Theta$ and "good" algebras $H \in \Theta$ lead to a geometry that is, in some sense, close to a traditional one.

Algebraic sets are defined by systems of equations $T$ of the type $w \equiv w^{\prime}$. Here we assume that there is a fixed finitely generated free algebra $W=W(X)$ in $\Theta$, and $w, w^{\prime}$ are elements of $W$.

Elementary sets are defined by systems $T$ of first order formulas. In this setting, arbitrary FOL formulas play a role occupied by the usual equations in the above setting.

Suppose that, along with a free algebra $W(X)$, we consider a special algebra of formulas $\Phi=\Phi(X)$. This $\Phi$ is also associated with the variety $\Theta$. Then a system of "equations" (= a system of first order formulas $T$ ) is a set of elements of $\Phi$. In particular, an equation $w=w^{\prime}$ is viewed as a formula of equality of the form $w \equiv w^{\prime}$, and this is an element in the algebra $\Phi(X)$.

Algebraic sets and elementary sets lie in the affine space $H^{X}$, which we identify in a standard way with the set of homomorphisms $\operatorname{Hom}(W(X), H)$. Hence, any point of an affine space is a homomorphism $\mu: W \rightarrow H$.

Let us consider the algebra $\Phi=\Phi(X)$ in more detail. We are not ready to give the precise definition of this algebra yet. It will be given in $\S 2$. For our immediate needs we say that $\Phi(X)$ is the algebra of compressed first order formulas, i.e., the quotient algebra of the usual first order formulas modulo their semantic equivalence. It is important to note the following.

1. $\Phi(X)$ is a Boolean algebra equipped with unary quantifier operations with respect to variables $x \in X$ and with equations $w \equiv w^{\prime}, w \in W(X)$, which are viewed as nullary operations.

2. With each formula $u \in \Phi$, its value $\operatorname{Val}_{H}^{X}(u)=A$ is associated, which is a subset in $\operatorname{Hom}(W(X), H)$. In particular,

$$
\operatorname{Val}_{H}^{X}\left(w \equiv w^{\prime}\right)=\left\{\mu: W \rightarrow H \mid\left(w, w^{\prime}\right) \in \operatorname{Ker}(\mu)\right\} .
$$

3. The logical kernel $\operatorname{LKer}(\mu)$ of a point $\mu: W \rightarrow H$ can be defined as follows. A formula $u \in \Phi(X)$ belongs to $\operatorname{LKer}(\mu)$ if and only if $\mu \in \operatorname{Val}_{H}(u)$. The usual kernel $\operatorname{Ker}(\mu)$ is the set of all $\left(w, w^{\prime}\right)$ with $w \equiv w^{\prime} \in \operatorname{LKer}(\mu)$. We say that a point $\mu$ is a solution of an "equation" $u$ if $u \in \operatorname{LKer}(\mu)$.

The logical kernel $\operatorname{LKer}(\mu)$ is an ultrafilter in the algebra $\Phi$. We denote by $M_{X}$ the set of all equalities $w \equiv w^{\prime}$ over the algebra $W=W(X)$. Then

$$
\operatorname{Ker}(\mu)=\operatorname{LKer}(\mu) \cap M_{X} .
$$

1.2. Galois correspondence. First, we recall this correspondence in universal algebraic geometry $[\mathrm{P} 1]-[\mathrm{P} 4]$. For more information on universal AG, see $[\mathrm{P} 1]-[\mathrm{P} 5]$ and P7, MR1, MR2].

Let $\Theta$ and a finitely generated free algebra $W(X) \in \Theta$ be fixed, and let $T$ be a binary relation on $W(X)$. We view $T$ as a system of equations $w \equiv w^{\prime},\left(w, w^{\prime}\right) \in T$, and as a system of formulas of the form $w \equiv w^{\prime}$. We define

$$
T_{H}^{\prime}=A=\{\mu: W \rightarrow H \mid T \subset \operatorname{Ker}(\mu)\}
$$

for every $H \in \Theta$.

We can write the definition of $T_{H}^{\prime}$ as

$$
T_{H}^{\prime}=A=\bigcap_{\left(w, w^{\prime}\right) \in T} \operatorname{Val}_{H}\left(w \equiv w^{\prime}\right) .
$$

Here $A$ is the set of points satisfying every equation in $T$, i.e., the set of solutions of all equations in $T$. Such sets $A$ are called algebraic sets. 
We define a correspondence in the reverse direction:

$$
A_{H}^{\prime}=T=\bigcap_{\mu \in A} \operatorname{Ker}(\mu)=\left\{\left(w, w^{\prime}\right) \mid A \subset \operatorname{Val}_{H}^{X}\left(w \equiv w^{\prime}\right)\right\} .
$$

Here $T$ is a congruence in $W$ called an $H$-closed congruence.

The resulting correspondence between algebraic sets and $H$-closed congruences is a Galois correspondence. This means that

1) $A_{1} \subset A_{2}$ implies $A_{2 H}^{\prime} \subset A_{1 H}^{\prime}$;

2) $T_{1} \subset T_{2}$ implies $T_{2 H}^{\prime} \subset T_{1 H}^{\prime}$;

3) $A \subset A_{H}^{\prime \prime}, T \subset T_{H}^{\prime \prime}$.

Here, the algebraic set $A_{H}^{\prime \prime}$ and the $H$-closed congruence $T_{H}^{\prime \prime}$ are the closures of a set $A$ and a system $T$, respectively.

Proposition 1.1. A congruence $T$ is $H$-closed if and only if $W / T \in S C(H)$.

Here $S$ is the operator of taking subgroups, while $C$ takes Cartesian products.

For each set of formulas $T$, consider quasi-identities of the form

$$
\bigwedge_{\left(w, w^{\prime}\right) \in T} w \equiv w^{\prime} \rightarrow w_{0} \equiv w_{0}^{\prime}
$$

or, briefly, $T \rightarrow w_{0} \equiv w_{0}^{\prime}$.

The set $T$ is not necessarily finite, and the formulas above are considered in the infinitary logic.

Proposition 1.2. We have $\left(w_{0}, w_{0}^{\prime}\right) \in T_{H}^{\prime \prime}$ if and only if the formula $T \rightarrow w_{0} \equiv w_{0}^{\prime}$ holds in $H$.

In logical geometry we start with the algebra of formulas $\Phi(X)$ and consider an arbitrary subset $T$ in $\Phi$. In this case, in order to establish a correspondence similar to the previous one, we shall replace the $\operatorname{kernel} \operatorname{Ker}(\mu)$ by the logical kernel $\operatorname{LKer}(\mu)$. We define

$$
T_{H}^{L}=A=\{\mu: W \rightarrow H \mid T \subset \operatorname{LKer}(\mu)\}=\bigcap_{u \in T} \operatorname{Val}_{H}(u) .
$$

Here $A$ is an elementary set in $\operatorname{Hom}(W, H)$ that consists of all points $\mu$ satisfying every "equation" $u \in T$. In the reverse direction:

$$
A_{H}^{L}=T=\bigcap_{\mu \in A} \operatorname{LKer}(\mu)=\left\{u \in \Phi\left(X \mid A \subset \operatorname{Val}_{H}^{X}(u)\right\} .\right.
$$

We defined the Galois correspondence in the case of logical geometry. The Galois closures are $A_{H}^{L L}$ and $T_{H}^{L L}$, respectively. Here $T=A_{H}^{L}$ is always an $H$-closed Boolean filter in $\Phi$.

For a given set of formulas $T \subset \Phi(X)$ and a given $v \in \Phi(X)$, consider the formula

$$
\bigwedge_{u \in T} u \rightarrow v
$$

or equally $T \rightarrow v$, where $T$ is not necessarily finite.

Proposition 1.3. We have $u \in T_{H}^{L L}$ if and only if the formula $T \rightarrow v$ holds in $H$.

1.3. Geometrical equivalence and logical equivalence of algebras. We recall (see [P1, P2]) that the algebras $H_{1}$ and $H_{2}$ are geometrically equivalent (AG-equivalent for short) if for every finite $X$ and $T$ in $W=W(X)$ we have

$$
T_{H_{1}}^{\prime \prime}=T_{H_{2}}^{\prime \prime} \text {. }
$$

See the survey $\mathrm{P} 3$ and $\mathrm{P} 2$ for the details.

Now we are able to define the notion of logically equivalent algebras. 
Definition 1.4. The algebras $H_{1}$ and $H_{2}$ are logically equivalent (LG-equivalent for short) if for every finite $X$ and $T$ in $\Phi=\Phi(X)$ we have

$$
T_{H_{1}}^{L L}=T_{H_{2}}^{L L} .
$$

We look at the idea of LG-equivalence from yet another point of view.

We denote by $\widehat{T}(H)$ the elementary theory of the algebra $H$. In accordance with the definition, a formula $u$ belongs to $\widehat{T}(H)$ if and only if $u$ is fulfilled in $H$ identically.

By definition, the algebras $H_{1}$ and $H_{2}$ are elementary equivalent if we have

$$
\widehat{T}\left(H_{1}\right)=\widehat{T}\left(H_{2}\right) .
$$

Consider formulas of the form

$$
u_{1} \wedge u_{2} \wedge \cdots \wedge u_{n} \rightarrow v,
$$

i.e., formulas $T_{0} \rightarrow v$, where $T_{0}$ is a finite set. Let $L G^{0}-\widehat{T}(H)$ denote the set of all formulas of this kind that hold in $H$. It is easily seen that $H_{1}$ and $H_{2}$ are elementary equivalent if and only if $L G^{0}-\widehat{T}\left(H_{1}\right)=L G^{0}-\widehat{T}\left(H_{2}\right)$.

Along with the invariants $\widehat{T}(H)$ and $L G^{0}-\widehat{T}(H)$ of the algebra $H$, consider the invariant $L G-\widehat{T}(H)$. We call $L G-\widehat{T}(H)$ the implicative theory of the algebra $H$. It consists of all formulas of the form

$$
T \rightarrow v
$$

that hold in $H$. Here, $T$ is a set of formulas in $\Phi$ (possibly infinite), and $v$ is a formula in $\Phi$.

Proposition 1.5. The algebras $H_{1}$ and $H_{2}$ are logically equivalent if and only if their implicative theories coincide, i.e.,

$$
L G-\widehat{T}\left(H_{1}\right)=L G-\widehat{T}\left(H_{2}\right) .
$$

This implies the following statement.

Proposition 1.6. If the algebras $H_{1}$ and $H_{2}$ are $L G$-equivalent, then they are elementary equivalent.

The reverse implication is not true, and hence, the relation of LG-equivalence is stronger than that of elementary equivalence.

The next statement is also clear.

Proposition 1.7. If the algebras $H_{1}$ and $H_{2}$ are $L G$-equivalent, then they are geometrically equivalent.

Definition 1.8. The algebras $H_{1}$ and $H_{2}$ are said to be weakly LG-equivalent if

$$
T_{H_{1}}^{L L}=T_{H_{2}}^{L L}
$$

for all finite $T$.

Proposition 1.9. The algebras $H_{1}$ and $H_{2}$ are weakly $L G$-equivalent if and only if they are elementary equivalent.

1.4. Noetherian-type properties. We shall consider conditions that provide the coincidence of the notions of LG-equivalence and elementary equivalence.

Definition 1.10. We say that an algebra $H \in \Theta$ is LG-Noetherian if for any elementary set $A=T_{H}^{L}$ there is an element $u$ in $\Phi=\Phi(X)$ such that $A=\operatorname{Val}_{H}^{X}(u)$.

In other words, this means that every elementary set is one-definable. 
Definition 1.11. An algebra $H \in \Theta$ is strongly LG-Noetherian if for every elementary set $A=T_{H}^{L}$ there is a finite subset $T_{0} \subset T$ such that $T_{H}^{L}=T_{0 H}^{L}$.

If $T_{0}=\left\{u_{1}, \ldots, u_{n}\right\}$, then $A$ is also one-definable by the element $u=u_{1} \wedge u_{2} \wedge \cdots \wedge u_{n}$. This element may fail to be an element of $T$.

Definition 1.12. An algebra $H \in \Theta$ is $H$-weakly LG-Noetherian if for every formula $T \rightarrow v \in L G-\widehat{T}(H)$ there is a finite subset $T_{0} \subset T$ such that $T_{0} \rightarrow v \in L G-\widehat{T}(H)$.

Note that here $T_{0}$ may depend on $v$.

Proposition 1.13. Suppose that the algebras $H_{1}$ and $H_{2}$ possess any of the Noetherian properties. The LG-equivalence of these algebras coincides with their elementary equivalence.

See $\S 3$ for the details.

1.5. Some categories and lattices. We fix an infinite set $X^{0}$ and let $\Gamma^{0}$ denote the set of all finite subsets of $X^{0}$.

For a given variety $\Theta$, denote by $\Theta^{0}$ the category whose objects are the free algebras $W=W(X)$ in $\Theta$ with finite $X \in \Gamma^{0}$. The category $\Theta^{0}$ is a full subcategory in the category $\Theta$. Its morphisms are homomorphisms of free algebras.

Given an algebra $H \in \Theta$, we define the category of affine spaces $K_{\Theta}^{0}(H)$ over $H$. Its objects are the sets of homomorphisms $\operatorname{Hom}(W, H), W \in \Theta^{0}$, and the morphisms are of the form

$$
\tilde{s}: \operatorname{Hom}\left(W_{1}, H\right) \rightarrow \operatorname{Hom}\left(W_{2}, H\right),
$$

where $s: W_{2} \rightarrow W_{1}$ is a morphism in $\Theta^{0}$ and the mapping $\tilde{s}$ is given by the rule $\tilde{s}(\nu)=\nu s: W_{2} \rightarrow H$ for $\nu: W_{1} \rightarrow H$. We have a contravariant functor $\Theta^{0} \rightarrow K_{\Theta}^{0}(H)$, which implies duality of the categories if and only if the identities of the algebra $H$ determine the entire variety $\Theta$, i.e., $\operatorname{Var}(H)=\Theta$ (see [P4]).

The next category is the category $\operatorname{Set}_{\Theta}(H)$ of affine sets over an algebra $H$. Its objects are of the form $(X, A)$, where $A$ is an arbitrary subset in the affine space $\operatorname{Hom}(W(X), H)$. The morphisms are

$$
[s]:(X, A) \rightarrow(Y, B) .
$$

Here $s: W(Y) \rightarrow W(X)$ is a morphism in $\Theta^{0}$. The corresponding $\tilde{s}: \operatorname{Hom}(W(X), H) \rightarrow$ $\operatorname{Hom}(W(Y), H)$ should be coordinated with $A$ and $B$ by the following condition: if $\nu \in A \subset \operatorname{Hom}(W(X), H)$, then $\tilde{s}(\nu) \in B \subset \operatorname{Hom}(W(Y), H)$. Then we view the induced mapping $[s]: A \rightarrow B$ as a morphism $(X, A) \rightarrow(Y, B)$.

Now we define the category of algebraic sets $K_{\Theta}(H)$ and the category of elementary sets $L K_{\Theta}(H)$. Both these categories are full subcategories in $\operatorname{Set}_{\Theta}(H)$ and are viewed as important invariants of the algebra $H$. We call them AG and LG-invariants of $H$.

The objects of the category $K_{\Theta}(H)$ are of the form $(X, A)$, where $A$ is an algebraic set in $\operatorname{Hom}(W(X), H)$. If we take the elementary sets for $A$, then we arrive at the category of elementary sets $L K_{\Theta}(H)$. The category $K_{\Theta}(H)$ is a full subcategory in $L K_{\Theta}(H)$.

Let us turn to the lattices. We shall see that if $A$ and $B$ are elementary sets in $\operatorname{Hom}(W, H)$, then the union $A \cup B$ is also an elementary set. This means that the elementary sets in $\operatorname{Hom}(W, H)$ constitute a lattice, which is a sublattice in the lattice of all subsets in the given affine space. A similar fact is not true in AG. For two algebraic sets $A$ and $B$, the set $A \cup B$ may fail to be an algebraic set. (Clearly, $A \cup B$ is an elementary set.)

In the sequel we shall coordinate the categories and lattices introduced above. 
1.6. Coordinate algebras and the categories of coordinate algebras. First, we look at the situation in AG. Let $A \subset \operatorname{Hom}(W, H)$ be an algebraic set, and let $T=A_{H}^{\prime}$ be an $H$-closed congruence in $W$. Then $W / T$ is called the coordinate algebra for $A$. It is an algebraic invariant of the algebraic set $A$. Denote by $C_{\Theta}(H)$ the category of coordinate algebras. It is a full subcategory in $\Theta$. It can be seen (consult [P2]) that there is a duality $K_{\Theta}(H) \rightarrow C_{\Theta}(H)$.

Now, we look at the same subject from the viewpoint of logical geometry. Let $A \subset$ $\operatorname{Hom}(W, H)$ be an elementary set and $T=A_{H}^{L}$ the corresponding Boolean filter in $\Phi(X)$. We have a Boolean algebra $\Phi(X) / T$, which is viewed as the coordinate algebra for $A$. The category of such coordinate Boolean algebras $L C_{\Theta}(H)$ will be defined in $\S 3$. The transition $(X, A) \rightarrow \Phi(X) / A_{H}^{L}$ determines a contravariant functor $L K_{\Theta}(H) \rightarrow L C_{\Theta}(H)$. However, this functor is not a duality in the general case.

Consider a particular example $A=\operatorname{Hom}(W(X), H)$. The definitions show that $A_{H}^{L}=$ $\widehat{T}^{X}(H)$, where $\widehat{T}^{X}(H)$ is the $X$ part of the elementary theory $\widehat{T}(H)$. At the same time, $\widehat{T}^{X}(H)$ is the minimal $H$-closed filter in $\Phi(X)$. The corresponding coordinate algebra for $A=\operatorname{Hom}(W, H)$ is the Boolean algebra $\Phi(X) / T h^{X}(H)$.

1.7. Algebras with the same logic. First, we recall some general facts from category theory; see $[\mathrm{M}]$. Let $\varphi_{1}, \varphi_{2}$ be two functors $C_{1} \rightarrow C_{2}$. An isomorphism $s: \varphi_{1} \rightarrow \varphi_{2}$ of functors is defined by the following conditions.

1. To every object $A$ of the category $C_{1}$, an isomorphism $s_{A}: \varphi_{1}(A) \rightarrow \varphi_{2}(A)$ in $C_{2}$ is assigned.

2. If $\nu: A \rightarrow B$ is a morphism in $C_{1}$, then there is a commutative diagram of the following form in $C_{2}$ :

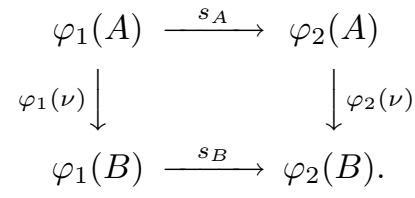

The isomorphism of functors $\varphi_{1}$ and $\varphi_{2}$ is denoted by $\varphi_{1} \simeq \varphi_{2}$. Now, let a pair $(\varphi, \psi)$ of functors $\varphi: C_{1} \rightarrow C_{2}$ and $\psi: C_{2} \rightarrow C_{1}$ be given. We say that it determines a category equivalence of $C_{1}$ and $C_{2}$ if $\psi \varphi \simeq 1_{C_{1}}$ and $\varphi \psi \simeq 1_{C_{2}}$. Here, $1_{C_{1}}$ and $1_{C_{2}}$ are identity functors. The conditions $\psi \varphi=1_{C_{1}}$ and $\varphi \psi=1_{C_{2}}$ define an isomorphism of categories. If $C_{1}=C_{2}=C$, then we get the notions of automorphism and autoequivalence of the category $C$.

An automorphism $\varphi$ of the category $C$ is said to be inner if it is isomorphic to the identity automorphism $1_{C}$. The latter means that if $s: 1_{C} \rightarrow \varphi$ is an isomorphism of functors, then for every object $A$ of $C$ there is an isomorphism $s_{A}: A \rightarrow \varphi(A)$ such that the diagram

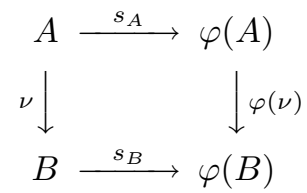

is commutative for any morphism $\nu: A \rightarrow B$ in $C$. So, $\varphi$ is inner if and only if it can be represented in the form

$$
\varphi(\nu)=s_{B} \nu s_{A}^{-1}: \varphi(A) \rightarrow \varphi(B) .
$$

This formula motivates the term "inner automorphism".

In the subsequent sections we shall consider the category $\mathrm{Hal}_{\Theta}^{0}$ of all algebras of formulas $\Phi(X)$, where $X$ runs through all finite subsets of $X^{0}$. In logical geometry, this category plays a role similar to that of the category of free algebras $\Theta^{0}$ in the case of 
algebraic geometry. We shall see that an automorphism $\varphi^{*}$ of $\mathrm{Hal}_{\Theta}^{0}$ corresponds to an automorphism $\varphi$ of $\Theta^{0}$. In particular, if $\varphi$ is inner, then $\varphi^{*}$ is also inner.

Now we consider the main topic of this subsection. Let Lat $_{H}: \Theta^{0} \rightarrow$ Lat be the functor that assigns to each $W(X)$ the lattice of elementary sets in $\operatorname{Hom}(W(X)), H)$. We say that the algebras $H_{1}$ and $H_{2}$ have the same lattices if there exists an automorphism $\varphi: \Theta^{0} \rightarrow \Theta^{0}$ such that the functors $\operatorname{Lat}_{H_{1}}$ and $\operatorname{Lat}_{H_{2}} \varphi$ are isomorphic. The coincidence of lattices can be represented by the commutative diagram

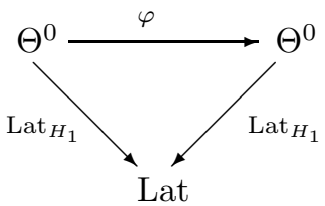

Here Lat is the category of lattices, and commutativity means the existence of an isomorphism Lat $H_{1} \rightarrow \operatorname{Lat}_{H_{2}} \varphi$ (see $\S 3$ ).

Consider also isomorphisms of categories $L K_{\Theta}\left(H_{1}\right) \rightarrow L K_{\Theta}\left(H_{2}\right)$. We require that there should exist a special correct isomorphism of these categories. Informally speaking, correctness means coordination of the category isomorphism with the lattices. We regard the algebras $H_{1}$ and $H_{2}$ as having the same logic if the categories $L K_{\Theta}\left(H_{1}\right)$ and $L K_{\Theta}\left(H_{2}\right)$ are correctly isomorphic.

This approach repeats similar definitions in the case of AG. In AG we prove that if $H_{1}$ and $H_{2}$ are AG-equivalent, then the categories $K_{\Theta}\left(H_{1}\right)$ and $K_{\Theta}\left(H_{2}\right)$ are correctly isomorphic. Similarly, the following is true.

Theorem 1.14. If the algebras $H_{1}$ and $H_{2}$ are $L G$-equivalent, then the categories $L K_{\Theta}\left(H_{1}\right)$ and $L K_{\Theta}\left(H_{2}\right)$ are correctly isomorphic.

(See Subsection 3.3 for the proof.) Here, LG-equivalence means that the corresponding logics are the same. In the case of $\mathrm{AG}$, the proof of a theorem of such a kind is trivial and is based on the duality between $K_{\Theta}(H)$ and $C_{\Theta}(H)$. In the case of LG there is no such duality and the proof is not trivial (see $\S 3$ ).

Note that the definition of the coincidence of logics can be grounded also on the correct equivalence of the categories of algebraic and elementary sets.

1.8. Some problems. We shall provide the reader with a list of problems related to the general scheme described above.

Problem 1.15. Consider various nonisomorphic LG-equivalent algebras.

It is hard to believe that LG-equivalence always implies isomorphism. Moreover, the general model-theoretic methods make it possible to construct nonisomorphic LGequivalent algebras. We are mostly interested in the cases where LG-equivalence of algebras implies their isomorphism. With this in mind, we introduce the following definitions.

Definition 1.16. Let $H_{1}$ and $H_{2}$ be two nonisomorphic algebras in $\Theta$. We say that they are LG-separated if they are not LG-equivalent.

Definition 1.17. An algebra $H$ is LG-separable in $\Theta$ if $H$ is not LG-equivalent to any other algebra $H^{\prime}$ in $\Theta$.

Problem 1.18. Consider varieties $\Theta$ such that every free algebra $W(X)$ in $\Theta$ is separable.

It is known (see Subsection 3.9) that the varieties of semigroups and inverse semigroups possess this property. There are also other examples of such a kind. 
Problem 1.19. What is the situation in the case of the variety of all groups, i.e., $\Theta=\operatorname{Grp} ?$

It can be proved (Z. Sela, unpublished) that every free group $F_{n}$ can be separated from the other free groups $F_{m}, n \neq m$. Hence, the question is what can be said if the second group is not free (see also Subsection 3.9).

Problem 1.20. What is the situation in the case of the variety of all commutative and associative algebras over a field $P$, i.e., $\Theta=\operatorname{Com}-P$ ?

Problem 1.21. What is the situation in the case of the variety of all associative algebras over a field $P$, i.e., $\Theta=A s s-P$, or in the case of the variety of all Lie algebras over a field $P$, i.e., $\Theta=$ Lie $-P$ ?

Problem 1.22. Let a free algebra $W(X)$ be fixed in the variety $\Theta$. Describe all algebras $H$ LG-equivalent to $W(X)$. In particular, consider $\Theta=$ Grp.

Problem 1.23. Let $H_{1}$ and $H_{2}$ be two Abelian groups. Suppose that they are LGequivalent. Is it true that they are isomorphic?

It can happen that this question has a positive answer.

Problem 1.24. Let $L_{1}$ and $L_{2}$ be two extensions of a field $P$. Suppose they are LGequivalent. Is it true that they are isomorphic?

In fact, a negative answer to this question in the case of arbitrary $L_{1}$ and $L_{2}$ can also be deduced from model theory (see the survey $G$ and the references therein). The most interesting case is to consider extensions with some natural restrictions on $L_{1}$ and $L_{2}$.

Now we mention the following important question. Let $H_{1}$ and $H_{2}$ be elementary equivalent. When does a single formula of the form $T \rightarrow v$ make $H_{1}$ and $H_{2}$ isomorphic? Or, vice versa, when can we separate nonisomorphic $H_{1}$ and $H_{2}$ with the help of a single formula of the form $T \rightarrow v$ ?

The following three problems are related to Noetherian algebras. It is clear that every finite algebra is LG-Noetherian.

Problem 1.25. Construct examples of infinite LG-Noetherian algebras.

Problem 1.26. Construct examples of weakly LG-Noetherian algebras.

Problem 1.27. Consider specific varieties $\Theta$ in respect to the properties of being LGNoetherian and weakly LG-Noetherinian. In particular, what is the situation for the variety of Abelian groups?

In the sequel we point out some other problems.

\section{§2. About ALGEBraiC LOGIC}

We always relate logic and algebraic logic to a fixed variety $\Theta$ of algebras.

Polyadic Halmos algebras and cylindric Tarski algebras are the main structures of algebraic logic [HMT, $\mathrm{H}]$. They used to be defined for an infinite set $X^{0}$ of variables. For our purposes we need to explore another situation, where we take the set $\Gamma^{0}$ of all finite subsets of $X^{0}$ instead of one infinite $X^{0}$. In particular, this leads to Halmos categories and special multisorted Halmos algebras. Here $\Gamma^{0}$ takes the role of the set of sorts. From now on $X$ denotes a finite set. 
2.1. Extended Boolean algebras. First, note that in algebraic logic (AL) quantifiers are treated as operations on Boolean algebras. Let $B$ be a Boolean algebra. Its existential quantifier is a mapping $\exists: B \rightarrow B$ that satisfies the following conditions:

1) $\exists 0=0$;

2) $\exists a>a$

3) $\exists(a \wedge \exists b)=\exists a \wedge \exists b$.

The universal quantifier $\forall: B \rightarrow B$ is defined dually:

1) $\forall 1=1$;

2) $\forall a<a$;

3) $\forall(a \vee \forall b)=\forall a \vee \forall b$.

Here 0 and 1 are the zero and the unit of the algebra $B$ and $a, b$ are arbitrary elements of $B$. The quantifiers $\exists$ and $\forall$ are coordinated in the usual way: $\overline{\exists a}=\forall \bar{a}, \overline{\forall a}=\exists \bar{a}$.

Let $\Theta$ and $W=W(X) \in \Theta$ be fixed, and let $B$ be a Boolean algebra. We call $B$ an extended Boolean algebra in $\Theta$ over $W(X)$ if

1. The quantifiers $\exists x$ for all $x \in X$ are defined in $B$ with $\exists x \exists y=\exists y \exists x$ for all $x, y \in X$.

2. With every formula $w \equiv w^{\prime}, w, w^{\prime} \in W$, a constant in $B$, denoted also by $w \equiv w^{\prime}$, is associated. Here,

2.1) $w \equiv w$ is the unit of the algebra $B$;

2.2 ) for every $n$-ary operation $\omega \in \Omega$ we have

$$
w_{1} \equiv w_{1}^{\prime} \wedge \cdots \wedge w_{n} \equiv w_{n}^{\prime}<w_{1} \cdots w_{n} \omega \equiv w_{1}^{\prime} \cdots w_{n}^{\prime} \omega .
$$

We can consider the variety of such algebras for given $\Theta$ and $W=W(X)$.

2.2. Example. Take an affine space $\operatorname{Hom}(W(X), H)$, and let

$$
\operatorname{Bool}(W(X), H)=\operatorname{Sub}(\operatorname{Hom}(W(X), H))
$$

be the Boolean algebra of all subsets $A$ in $\operatorname{Hom}(W(X), H)$. We define quantifiers $\exists x$, $x \in X$, on the algebra $\operatorname{Bool}(W(X), H)$. We set $\mu \in \exists x A$ if and only if there exists $\nu \in A$ such that $\mu(y)=\nu(y)$ for every $y \in X, y \neq x$.

Every equality $w \equiv w^{\prime}, w, w^{\prime} \in W$, is implemented on this algebra as

$$
\operatorname{Val}_{H}^{X}\left(w \equiv w^{\prime}\right)=\left\{\mu: W \rightarrow H \mid\left(w, w^{\prime}\right) \in \operatorname{Ker}(\mu)\right\} .
$$

As a result, we have an extended $\operatorname{Bool}(W(X), H)$ in $\Theta$ over $W(X)$.

Next, consider the category $\operatorname{Hal}_{\Theta}(H)$ of extended Boolean algebras for a given $H \in \Theta$. Its morphisms are of the form

$$
s_{*}: \operatorname{Bool}(W(X), H) \rightarrow \operatorname{Bool}(W(Y), H),
$$

where $s: W(X) \rightarrow W(Y)$ is a morphism in $\Theta^{0}$. We define the transition from $s$ to $s_{*}$. We have

$$
\tilde{s}: \operatorname{Hom}(W(Y), H) \rightarrow \operatorname{Hom}(W(X), H) .
$$

Let $A$ be a subset in $\operatorname{Hom}(W(X), H)$. We set $s_{*} A=\tilde{s}^{-1} A$. The map $s_{*}$ is a homomorphism of Boolean algebras, but, in general, not a homomorphism of extended Boolean algebras.

We have a covariant functor $\Theta^{0} \rightarrow \operatorname{Hal}_{\Theta}(H)$.

2.3. Halmos categories. A category $\Upsilon$ is a Halmos category if the following is true.

1. Every object of $\Upsilon$ has the form $\Upsilon(X)$, and this object is an extended Boolean algebra in $\Theta$ over $W(X)$.

2. The morphisms are of the form $s_{*}: \Upsilon(X) \rightarrow \Upsilon(Y)$, where the $s: W(X) \rightarrow W(Y)$ are morphisms in $\Theta^{0}$, the $s_{*}$ are homomorphisms of Boolean algebras, and the transition $s \rightarrow s_{*}$ is given by a covariant functor $\Theta^{0} \rightarrow \Upsilon$. 
3. There are identities controlling the interaction of morphisms with quantifiers and equalities. The coordination with the quantifiers is as follows:

3.1) $s_{1 *} \exists x a=s_{2 *} \exists x a, \quad a \in \Upsilon(X)$, if $s_{1} y=s_{2} y$ for every $y \in X, y \neq x$.

3.2) $s_{*} \exists x a=\exists(s x)\left(s_{*} a\right)$ if $s x=y \in Y$ and $y=s x$ is not in the support of $s x^{\prime}$, $x^{\prime} \in X, x^{\prime} \neq x$.

4. The following conditions describe the coordination with equalities:

4.1) $s_{*}\left(w \equiv w^{\prime}\right)=\left(s w \equiv s w^{\prime}\right)$ for $s: W(X) \rightarrow W(Y), w, w^{\prime} \in W(X)$.

4.2) $s_{w}^{x} a \wedge\left(w \equiv w^{\prime}\right)<s_{w^{\prime}}^{x} a$ for an arbitrary $a \in \Upsilon(X), x \in X$, where $w, w^{\prime} \in$ $W(X)$, and $s_{w}^{x}: W(X) \rightarrow W(X)$ is defined by the rule $s_{w}^{x}(x)=w, s_{w}^{x}(y)=y, y \in X$, $y \neq x$.

The category $\operatorname{Hal}_{\Theta}(H)$ is an example of a Halmos category. Another important example is the category of formulas $\mathrm{Hal}_{\Theta}^{0}$ of the algebras of formulas $\mathrm{Hal}_{\Theta}^{0}(X)=\Phi(X)$. As was mentioned before, in logical geometry this category plays the same role as the category $\Theta^{0}$ plays in AG.

2.4. Multisorted algebras. We shall use multisorted algebras in order to define the notion of Halmos algebras. One-sorted algebras are algebras with one domain. In multisorted algebras there are many domains. They are written as $G=\left(G_{i}, i \in \Gamma\right)$, where $\Gamma$ is a set of sorts, which can be infinite. Categories are often related to multisorted algebras [Hi].

Every operation $\omega$ in $G$ has a specific type $\eta=\eta(\omega)$. In the one-sorted case it is the arity of an operation. In the multisorted case we have $\eta=\left(i_{1}, \ldots, i_{n} ; j\right)$ and a mappingoperation $\omega: G_{i_{1}} \times \cdots \times G_{i_{n}} \rightarrow G_{j}$. Morphisms of multisorted algebras are of the form $\mu=\left(\mu_{i}, i \in \Gamma\right): G \rightarrow G^{\prime}$, where the $\mu_{i}: G_{i} \rightarrow G_{i}^{\prime}$ are the mappings and $\mu$ is naturally correlated with the operations $\omega$.

Subalgebras, quotient algebras, and Cartesian products of multisorted algebras are defined in the usual way. Hence, one can define varieties of multisorted algebras with a given domain $\Gamma$ and signature $\Omega$. In every such variety there exist free algebras determined by multisorted sets (see also $\mathrm{Hi}$ ).

2.5. Halmos algebras. We deal with multisorted Halmos algebras associated with Halmos categories. First, we define the signature $L_{X}$. Take $L_{X}=\left\{\vee, \wedge,{ }^{-}, \exists x, x \in X, M_{X}\right\}$ for every $X$. Here $M_{X}$ is the set of all equalities over the algebra $W=W(X)$. We add all $s: W(X) \rightarrow W(Y)$ to $L_{X}$, treating them as symbols of unary operations. The new signature is denoted by $L_{\Theta}$.

Next, we consider algebras $\Upsilon=\left(\Upsilon_{X}, X \in \Gamma\right)$. Every $\Upsilon_{X}$ is an algebra in the signature $L_{X}$, and a unary operation (mapping) $s_{*}: \Upsilon_{X} \rightarrow \Upsilon_{Y}$ corresponds to every $s: W(X) \rightarrow$ $W(Y)$. An algebra $\Upsilon$ in the signature $L_{\Theta}$ will be called a Halmos algebra if

1) every $\Upsilon_{X}$ is an extended Boolean algebra in the signature $L_{X}$;

2) every mapping $s_{*}: \Upsilon_{X} \rightarrow \Upsilon_{Y}$ is coordinated with the Boolean operations and is a homomorphism of Boolean algebras;

3 ) the identities controlling interaction of the operations $s_{*}$ with quantifiers and equalities are the same as in the definition of Halmos categories.

Now it is clear that each Halmos category $\Upsilon$ can be viewed as a Halmos algebra and vice versa. In particular, this is true for $\operatorname{Hal}_{\Theta}(H)$.

2.6. Categories and algebras of formulas. Denote by $M=\left(M_{X}, X \in \Gamma\right)$ a multisorted set with the components $M_{X}$.

Let $\Upsilon^{0}=\left(\Upsilon_{X}^{0}, X \in \Gamma^{0}\right)$ be the absolutely free algebra over $M$ in the signature $L_{\Theta}$. The elements of each $\Upsilon_{X}^{0}$ are first order logic (FOL) formulas, which are constructed inductively from the equalities by using the signature $L_{\Theta}$. So, $\Upsilon^{0}$ is a multisorted algebra of pure FOL formulas over equalities. 
Let $\mathrm{Hal}_{\Theta}$ denote the variety of $\Gamma^{0}$-sorted Halmos algebras in the signature $L_{\Theta}$, and let $\mathrm{Hal}_{\Theta}^{0}$ be the free algebra of this variety over the multisorted set of equalities $M$. The same $M$ determines the homomorphism $\pi=\left(\pi_{X}, X \in \Gamma\right): \Upsilon^{0} \rightarrow \mathrm{Hal}_{\Theta}^{0}$. If $u \in \Upsilon_{X}^{0}$, then the image $u^{\pi} x=\bar{u}$ in $\operatorname{Hal}_{\Theta}^{0}(X)$ is viewed as a compressed formula.

Setting $\operatorname{Hal}_{\Theta}^{0}(X)=\Phi(X)$, we get the required algebra of compressed formulas. This is an extended Boolean algebra.

Recall that the Halmos algebra $\mathrm{Hal}_{\Theta}^{0}$ of formulas is also a Halmos category. We have a covariant functor $\Theta^{0} \rightarrow \mathrm{Hal}_{\Theta}^{0}$.

2.7. Value of a formula. The value $\operatorname{Val}_{H}^{X}\left(w \equiv w^{\prime}\right)$ corresponds to each equality $w \equiv w^{\prime}$ with $w, w^{\prime} \in W(X)$. This determines a mapping $\operatorname{Val}_{H}: M \rightarrow \operatorname{Hal}_{\Theta}(H)$, which is uniquely extended up to the homomorphisms $\operatorname{Val}_{H}^{0}: \Upsilon^{0} \rightarrow \operatorname{Hal}_{\Theta}(H)$ and $\operatorname{Val}_{H}: \operatorname{Hal}_{\Theta}^{0} \rightarrow \operatorname{Hal}_{\Theta}(H)$. For every $X \in \Gamma$ we have a commutative diagram

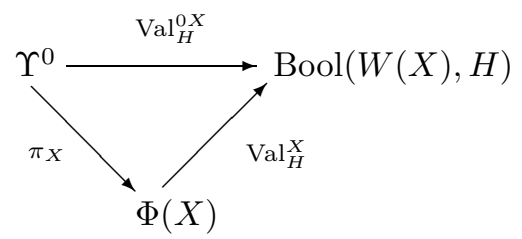

Thus, for every $u \in \Upsilon_{X}^{0}$ and the corresponding $\bar{u} \in \Phi(X)$ we have the values $\operatorname{Val}_{H}^{0 X}(u)=$ $\operatorname{Val}_{H}^{X}(\bar{u})$.

Formulas $u$ and $v$ in $\Upsilon_{X}^{0}$ are said to be semantically equivalent if $\operatorname{Val}_{\Theta}^{0}(u)=\operatorname{Val}_{\Theta}^{0}(v)$ for every algebra $H \in \Theta$. In $[\overline{\mathrm{P}} 6$ it was proved that

1) formulas $u$ and $v$ are semantically equivalent if and only if $u^{\pi_{X}}=\bar{u}=\bar{v}=v^{\pi_{X}}$;

2) the variety $\mathrm{Hal}_{\Theta}$ is generated by all algebras $\operatorname{Hal}_{\Theta}(H)$, where $H \in \Theta$.

The second proposition motivates the definition of the variety $\mathrm{Hal}_{\Theta}$ as a variety determined by common identities of all $\operatorname{Hal}_{\Theta}(H)$ for every $H \in \Theta$. These identities were specified in the definition of a Halmos category.

We make a remark on the kernel of the homomorphism $\mathrm{Val}_{H}$. We have

$$
\operatorname{Ker}\left(\operatorname{Val}_{H}\right)=\operatorname{Th}(H)=\left(\operatorname{Th}^{X}(H), X \in \Gamma\right) .
$$

Here $\operatorname{Th}(H)=\left(\operatorname{Th}^{X}(H), X \in \Gamma\right)$ is the elementary theory of the algebra $H$, i.e., the set of formulas $u \in \operatorname{Th}^{X}(H)$ such that $\operatorname{Val}_{H}^{X}(u)=\operatorname{Hom}(W(X), H)$ for every $X$. It is clear also that the image $\operatorname{Im~} \mathrm{Val}_{H}$ is a subalgebra in $\mathrm{Hal}_{\Theta}(H)$, which consists of one-defined elementary sets. This is the necessary information from algebraic logic.

\subsection{Variation of the variety $\Theta$.}

Proposition 2.1. The elementary equivalence of algebras and the logical equivalence of algebras are invariant with respect to the choice of the variety $\Theta$.

Let $H$ be an algebra in $\Theta$, and take a subvariety $\Theta_{1}$ in $\Theta$ that also contains $H$. Let $W=W(X)$ and $W^{1}=W^{1}(X)$ be the free algebras over the set $X$ in $\Theta$ and $\Theta_{1}$, respectively. We have the homomorphism $\pi_{X}: W(X) \rightarrow W^{1}(X)$ and the commutative diagram

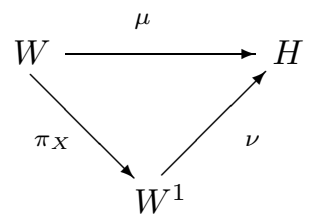


This diagram gives rise to the bijection $\widetilde{\pi}_{X}: \operatorname{Hom}\left(W^{1}, H\right) \rightarrow \operatorname{Hom}(W, H)$ defined by $\tilde{\pi}_{X}(\nu)=\mu=\nu \pi_{X}$. The same $\tilde{\pi}_{X}$ induces an isomorphism of Boolean algebras:

$$
\tilde{\pi}_{X}: \operatorname{Bool}\left(W^{1}, H\right) \rightarrow \operatorname{Bool}(W, H) .
$$

We shall coordinate this isomorphism with quantifiers and equalities. Let $A$ be a set of points $\nu: W^{1} \rightarrow H$, and let $x \in X$. We check the relation $\widetilde{\pi}_{X}(\exists x A)=\exists x\left(\widetilde{\pi}_{X}(A)\right)$.

Take $\mu \in \widetilde{\pi}_{X}(\exists x A), \mu=\widetilde{\pi}_{X}(\nu)=\nu \pi_{X}, \nu \in \exists x A$. There is $\nu_{1} \in A$ with $\nu(y)=\nu_{1}(y)$ for every $y \in X, y \neq x$. Next, let $\mu_{1}=\nu_{1} \pi_{X} \in \tilde{\pi}_{X}(A)$. We have

$$
\mu_{1}(y)=\nu_{1} \pi_{X}(y)=\nu_{1}(y)=\nu(y)=\nu \pi_{X}(y)=\mu(y),
$$

whence $\mu \in \exists x \widetilde{\pi}_{X}(A)$. Here we identify $\widetilde{\pi}_{X}(y)=y$, for every $y \in X$.

Let $\mu \in \exists x \widetilde{\pi}_{X}(A)$. We must check that there exists $\nu \in \exists x A$ such that $\mu=\nu \pi_{X}$. Take $\mu_{1} \in \widetilde{\pi}_{X}(A)$ with $\mu(y)=\mu_{1}(y)$ for $y \neq x$. Here, $\mu_{1}=\nu_{1} \pi_{X}$ for $\nu_{1} \in A$. We have

$$
\mu(y)=\mu_{1}(y)=\nu_{1} \pi_{X}(y)=\nu_{1}(y) .
$$

We take $\nu$ satisfying $\nu(y)=\nu_{1}(y)$ for every $y \neq x$ and $\nu(x)=\mu(x)$. Then $\nu \in \exists x A$ and always $\mu(x)=\nu(x)=\nu \pi_{X}(x), \mu=\nu \pi_{X}, \mu \in \widetilde{\pi}_{X}(\exists x A)$.

Also, we have

$$
\tilde{\pi}_{X}^{-1}(\exists x B)=\exists x\left(\widetilde{\pi}_{X}^{-1}(B)\right)
$$

for $B \subset \operatorname{Hom}(W(X), H)$.

Now we consider the coordination between $\pi_{X}$ and the equalities. Take an equality $w \equiv w^{\prime}, w, w^{\prime} \in W$. It corresponds to the equality $\bar{w} \equiv \overline{w^{\prime}}$ in $\Theta_{1}$, where $\bar{w}=\pi_{X}(w)$. Here, $w \equiv w^{\prime}$ is viewed as a symbol of equality which is implemented in $\Theta$ and $\Theta_{1}$. We take $\operatorname{Val}_{H}^{X}: \Phi(X) \rightarrow \operatorname{Bool}(W(X), H)$, and $\operatorname{Val}_{H}^{X}: \Phi^{1}(X) \rightarrow \operatorname{Bool}\left(W^{1}(X), H\right)$. Here, $\Phi^{1}(X)$ is the algebra of formulas for $\Theta_{1}$. We have

$$
\widetilde{\pi}_{X}\left(\operatorname{Val}_{H}^{\prime X}\left(\bar{w} \equiv \overline{w^{\prime}}\right)\right)=\operatorname{Val}_{H}^{X}\left(w \equiv w^{\prime}\right) .
$$

So, $\widetilde{\pi}_{X}$ is coordinated with the equalities, and we have isomorphisms of extended Boolean algebras:

$$
\tilde{\pi}_{X}: \operatorname{Bool}\left(W^{1}(X), H\right) \rightarrow \operatorname{Bool}(W(X), H)
$$

and

$$
\widetilde{\pi}_{X}^{-1}: \operatorname{Bool}(W(X), H) \rightarrow \operatorname{Bool}\left(W^{1}(X), H\right) .
$$

Now we relate the varieties $\mathrm{Hal}_{\Theta}$ and $\mathrm{Hal}_{\Theta_{1}}$ and the algebra-categories $\mathrm{Hal}_{\Theta}^{0}$ and $\operatorname{Hal}_{\Theta_{1}}^{0}$. The variety $\mathrm{Hal}_{\Theta}$ is generated by all algebras $\operatorname{Hal}_{\Theta}(H), H \in \Theta$. Consider a subvariety in $\mathrm{Hal}_{\Theta}$ generated by all algebras $\mathrm{Hal}_{\Theta}(H), H \in \Theta_{1}$. This is a subvariety in the signature $L_{\Theta}$. Proceeding from the signature $L_{\Theta_{1}}$, we get the variety coinciding with $\mathrm{Hal}_{\Theta_{1}}$. Thus, if $\Theta_{1}$ is a subvariety in $\Theta$, then $\mathrm{Hal}_{\Theta_{1}}$ is embedded in $\mathrm{Hal}_{\Theta}$.

We describe $\mathrm{Hal}_{\Theta}^{0}$ and $\mathrm{Hal}_{\Theta_{1}}^{0}$. The transition $w \equiv w^{\prime} \rightarrow \bar{w} \equiv \overline{w^{\prime}}$ determines a surjection $M_{X} \rightarrow M_{X}^{1}$, where $M_{X}^{1}$ is related to $\Theta_{1}$. Denote $M=\left(M_{X}, X \in \Gamma^{0}\right)$ and $M^{1}=$ $\left(M_{X}^{1}, X \in \Gamma^{0}\right)$. We have a surjective map $\tau=\left(\tau_{X}, X \in \Gamma^{0}\right): M \rightarrow M^{1}$. This $\tau$ can be extended to a homomorphism $\tau: \operatorname{Hal}_{\Theta}^{0} \rightarrow \operatorname{Hal}_{\Theta_{1}}^{0}$. For each $X \in \Gamma^{0}$ we have $\tau_{X}: \Phi(X) \rightarrow \Phi^{1}(X)$. Also, we have a commutative diagram

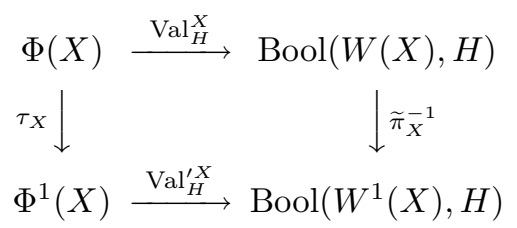

The commutativity of this diagram follows from the coordinations above. 
Now we consider the elementary theories $\widehat{T}_{\Theta}^{X}(H)$ and $\widehat{T}_{\Theta_{1}}^{X}(H)$. The diagram shows that $u \in \widehat{T}_{\Theta}^{X}(H)$ if and only if $\tau_{X}(u) \in \widehat{T}_{\Theta_{1}}^{X}(H)$. The latter implies that $\widehat{T}_{\Theta}^{X}\left(H_{1}\right)=\widehat{T}_{\Theta}^{X}\left(H_{2}\right)$ if and only if $\widehat{T}_{\Theta_{1}}^{X}\left(H_{1}\right)=\widehat{T}_{\Theta_{1}}^{X}\left(H_{2}\right)$.

Hence, the elementary equivalence of algebras is invariant with respect to the choice of the variety $\Theta$.

We check that this property is also fulfilled for LG-equivalent algebras.

Let $T$ be a set of formulas in $\Phi(X)$. Denote $T^{\tau_{X}}=\left\{\tau_{X}(u) u \mid u \in T\right\} \subset \Phi^{1}(X)$. For each formula $u \in T$, we have $\widetilde{\pi}_{X}^{-1}\left(\operatorname{Val}_{H}^{X}(u)\right)=\operatorname{Val}_{H}^{\prime X}\left(\tau_{X}(u)\right)$. Then

$$
\left(T^{\tau_{X}}\right)_{H}^{L}=\bigcap_{u \in T} \operatorname{Val}_{H}^{\prime X}\left(\tau_{X}(u)\right)=\bigcap_{u \in T} \tilde{\pi}_{X}^{-1}\left(\operatorname{Val}_{H}^{X}(u)\right)=\tilde{\pi}^{-1}\left(\bigcap_{u \in T} \operatorname{Val}_{H}^{X}(u)\right)=\tilde{\pi}_{X}^{-1} T_{H}^{L} .
$$

Now, let $u \in T_{H}^{L L}$. This means that $T_{H}^{L} \subset \operatorname{Val}_{H}^{X}(u)$. Then $\widetilde{\pi}_{X}^{-1}\left(T_{H}^{L}\right) \subset \widetilde{\pi}_{X}^{-1} \operatorname{Val}_{H}^{X}(u)$. This gives $\left(T^{\tau_{X}}\right)_{H}^{L} \subset \operatorname{Val}_{H}^{\prime X}\left(\tau_{X}(u)\right)$ and $\tau_{X}(u) \in\left(T^{\tau_{X}}\right)_{H}^{L L}$. The inverse embedding is also valid. Hence, $u \in T_{H}^{L L}$ if and only if $\tau_{X}(u) \in\left(T^{\tau_{X}}\right)_{H}^{L L}$.

Now, assuming that

$$
T_{H_{1}}^{L L}=T_{H_{2}}^{L L},
$$

we check the formula

$$
T_{H_{1}}^{\tau_{X} L L}=T_{H_{2}}^{\tau_{X} L L} .
$$

Let $\tau_{X}(u) \in T_{H_{1}}^{\tau_{X} L L}$. Then $u \in T_{H_{1}}^{L L}$ and $u \in T_{H_{2}}^{L L}$, i.e., $\tau_{X}(u) \in T_{H_{2}}^{\tau_{X} L L}$. The same is true in the reverse direction. Hence, $T_{H_{1}}^{L L}=T_{H_{2}}^{L L}$ if and only if $T_{H_{1}}^{\tau_{X} L L}=T_{H_{2}}^{\tau_{X}}$. ${ }^{L L}$ This means that $L G$-equivalence does not depend on the choice of the variety $\Theta$.

\section{§3. LOGICAL GEOMETRY}

In this section we continue the streamline outlined in $\S 1$. We shall use the material of $\S 2$, where the background from algebraic logic was given.

3.1. Lattices of elementary sets and $H$-closed filters. Let $A$ and $B$ be two elementary sets in $\operatorname{Hom}(W(X), H)$. We show that $A \cup B$ is also an elementary set.

Consider the filters $T_{1}=A_{H}^{L}$ and $T_{2}=B_{H}^{L}$. Denote by $T_{1} \vee T_{2}$ the set of all formulas of the form $u \vee v$, where $u \in T_{1}$ and $v \in T_{2}$. We have $T_{1} \vee T_{2} \subset T_{1} \cap T_{2}$. Then

$$
\left(T_{1} \vee T_{2}\right)_{H}^{L}=\bigcap_{T_{1} \vee T_{2}} \operatorname{Val}_{H}^{X}(u \vee v)=\left(\bigcap_{u \in T_{1}} \operatorname{Val}_{H}^{X}(u)\right) \cup\left(\bigcap_{v \in T_{2}} \operatorname{Val}_{H}^{X}(v)\right)=A \cup B .
$$

Hence, $\left(T_{1} \cap T_{2}\right)_{H}^{L}=A \cup B$.

This lattice will be denoted by $\operatorname{Lat}_{H}(\Phi(X))$. The lattice $\operatorname{Lat}_{H}^{*}(\Phi(X))$ of all $H$ closed filters in the algebra $\Phi(X)$ can also be considered. Given $X \in \Gamma^{0}$, both lattices $\operatorname{Lat}_{H}(\Phi(X))$ and $\operatorname{Lat}_{H}^{*}(\Phi(X))$ are anti-isomorphic distributive lattices. Consider two functors:

$$
\mathrm{LCl}_{H}: \mathrm{Hal}_{\Theta}^{0} \rightarrow \text { Lat }
$$

and

$$
\mathrm{LCl}_{H}^{*}: \mathrm{Hal}_{\Theta}^{0} \rightarrow \text { poSet. }
$$

Here Lat denotes the category of lattices and poSet is the category of partially ordered sets. The first functor is covariant, while the second is contravariant. We have $\mathrm{LCl}_{H}(\Phi(X))=\operatorname{Lat}_{H}(\Phi(X)), X \in \Gamma^{0}$, i.e., $\mathrm{LCl}_{H}(\Phi(X))$ is the lattice of all elementary sets in $\operatorname{Hom}(W(X), H)$. Similarly, $\operatorname{LCl}_{H}^{*}(\Phi(X))$ is the lattice of all $H$-closed filters in $\Phi(X)$. 
Let $s_{*}: \Phi(X) \rightarrow \Phi(Y)$ be a morphism in $\operatorname{Hal}_{\Theta}^{0}$. We have also $s: W(X) \rightarrow W(Y)$. Define

$$
\begin{aligned}
& \operatorname{LCl}_{H}\left(s_{*}\right): \operatorname{LCl}_{H}(\Phi(X)) \rightarrow \mathrm{LCl}_{H}(\Phi(Y)), \\
& \operatorname{LCl}_{H}^{*}\left(s_{*}\right): \operatorname{LCl}_{H}^{*}(\Phi(Y)) \rightarrow \mathrm{LCl}_{H}^{*}(\Phi(X)) .
\end{aligned}
$$

Take $A \subset \mathrm{LCl}_{H}(\Phi(X)), A \subset \operatorname{Hom}(W(X), H)$. We consider $\widetilde{s}: \operatorname{Hom}(W(Y), H) \rightarrow$ $\operatorname{Hom}(W(X), H)$ and take $\widetilde{s}^{-1} A=s_{*} A$. Denote $\mathrm{LCl}_{H}\left(s_{*}\right)(A)=s_{*} A$. The functor $\mathrm{LCl}_{H}$ is defined and coordinated with the lattice operations.

In order to define the functor $\mathrm{LCl}_{H}^{*}$, take $T \in \mathrm{LCl}_{H}^{*}(\Phi(Y))$. Then $s_{*} T$ is the set of all $u \in \Phi(X)$ such that $s_{*} u \in T$. We shall see that $s_{*} T$ is an $H$-closed filter in $\Phi(X)$. Defining $\mathrm{LCl}_{H}^{*}\left(s_{*}\right)(T)=s_{*} T$, we determine the functor $\mathrm{LCl}_{H}^{*}$.

Let $\varphi: \Theta^{0} \rightarrow \Theta^{0}$ be an automorphism of the category $\Theta^{0}$. It corresponds to $\varphi^{*}$ : $\operatorname{Hal}_{\Theta}^{0} \rightarrow \operatorname{Hal}_{\Theta}^{0}$ defined by the rule $\varphi^{*}(\Phi(X))=\Phi(Y)$ if $\varphi(W(X))=W(Y)$, and $\varphi^{*}\left(s_{*}\right)=$ $\varphi(s)_{*}: \varphi^{*}(\Phi(X)) \rightarrow \varphi^{*}(\Phi(Y))$ if $\varphi(s): \varphi(W(X)) \rightarrow \varphi(W(Y))$ is a morphism in $\Theta^{0}$.

Consider the product $\mathrm{LCl}_{H} \varphi^{*}$ and apply it to $\Phi(X)$. We get the lattice $\mathrm{LCl}_{H}(\Phi(Y))$ of all elementary sets in $\operatorname{Hom}(W(Y), H)=\operatorname{Hom}(\varphi(W(X)), H)$.

Suppose that there is an isomorphism of functors $\mathrm{LCl}_{H_{1}} \rightarrow \mathrm{LCl}_{H_{2}} \varphi^{*}$. Then for every $\Phi(X)$ there is an isomorphism of lattices $\mathrm{LCl}_{H_{1}}(\Phi(X)) \rightarrow \mathrm{LCl}_{H_{2}}(\Phi(Y))$. This means that there is an isomorphism of the lattices of elementary sets in $\operatorname{Hom}\left(W(X), H_{1}\right)$ and in $\operatorname{Hom}\left(\varphi(W(X)), H_{2}\right)$.

We say that the algebras $H_{1}$ and $H_{2}$ have the same lattices of elementary sets if there exists a functor $\varphi: \Theta^{0} \rightarrow \Theta^{0}$ such that there is an isomorphism of functors $\mathrm{LCl}_{H_{1}} \rightarrow \mathrm{LCl}_{H_{2}} \varphi^{*}$. An isomorphism $\mathrm{LCl}_{H_{1}}^{*} \rightarrow \mathrm{LCl}_{H_{2}}^{*} \varphi^{*}$ is used in a similar way.

In the sequel we shall consider these lattice isomorphisms and category isomorphisms $L K_{\Theta}\left(H_{1}\right) \rightarrow L K_{\Theta}\left(H_{2}\right)$ and $L C_{\Theta}\left(H_{1}\right) \rightarrow L C_{\Theta}\left(H_{2}\right)$ all together.

3.2. Correct isomorphism of categories of elementary sets. Our aim is to define the notion of a correct isomorphism of elementary sets. First, we note that the category of affine spaces $K_{\Theta}^{0}(H)$ is always a subcategory in the category of elementary sets $L K_{\Theta}(H)$.

Let an isomorphism $F: L K_{\Theta}\left(H_{1}\right) \rightarrow L K_{\Theta}\left(H_{2}\right)$ be given. Suppose that $F$ takes $K_{\Theta}^{0}\left(H_{1}\right)$ to $K_{\Theta}^{0}\left(H_{2}\right)$. This means that there is an automorphism $\varphi: \Theta^{0} \rightarrow \Theta^{0}$ such that $F\left(\operatorname{Hom}\left(W, H_{1}\right)\right)=\operatorname{Hom}\left(\varphi(W), H_{2}\right)$ for every object $W=W(X)$ in the category $\Theta^{0}$.

Next, let $(X, A)$ be an object in the category $L K_{\Theta}\left(H_{1}\right)$ and $F(X, A)=(Y, B)$. Here we assume that $W(Y)=\varphi(W(X))$ and $B$ is an elementary set in $\operatorname{Hom}\left(W(Y), H_{2}\right)$. Assume also that for every $W=W(X)$ the isomorphism $F$ induces an isomorphism of the lattices of elementary sets in $\operatorname{Hom}\left(W, H_{1}\right)$ and in $\operatorname{Hom}\left(\varphi(W), H_{2}\right)$.

An isomorphism $F: L K_{\Theta}\left(H_{1}\right) \rightarrow L K_{\Theta}\left(H_{2}\right)$ is said to be correct if it satisfies the conditions above. However, we cannot say that such an isomorphism always induces an isomorphism of lattices $L C l_{H_{1}} \rightarrow L_{C l} l_{H_{2}} \varphi^{*}$.

We finish this subsection with the remark that we can build a similar theory by replacing the notion of a correct isomorphism by a correct equivalence of the categories of elementary sets.

3.3. LG-equivalence of algebras implies a correct isomorphism of categories. In this section we prove Theorem 1.14. We start with some general remarks. Take $s: W(X) \rightarrow W(Y)$ and, accordingly, $s_{*}: \Phi(X) \rightarrow \Phi(Y)$. For $T \subset \Phi(Y)$ we set $u \in s_{*} T$ if $s_{*} u \in T$. For $T \subset \Phi(X)$ we have $s^{*} T=\left\{s_{*} u \mid u \in T\right\}$. Further, $\tilde{s}$ : $\operatorname{Hom}(W(Y), H) \rightarrow \operatorname{Hom}(W(X), H)$. Take $B=s_{*} A=\tilde{s}^{-1} A$ for $A \subset \operatorname{Hom}(W(X), H)$. For $B \subset \operatorname{Hom}(W(Y), H)$ we have $s^{*} B=\{\tilde{s}(\mu) \mid \mu \in B\}$.

We have the following properties:

1) if $T \subset \Phi(X)$, then $\left(s^{*} T\right)_{H}^{L}=s_{*} T_{H}^{L}$; 
2) if $B \subset \operatorname{Hom}(W(Y), H)$, then $\left(s^{*} B\right)_{H}^{L}=s_{*} B_{H}^{L}$;

3) if $A \subset \operatorname{Hom}(W(X), H)$, then $s^{*} A_{H}^{L} \subset\left(s_{*} A\right)_{H}^{L}$.

We view these properties as rules of the behavior of elementary sets under the moves of affine spaces. The first rule implies that if $A$ is an elementary set, then so is $s_{*} A$. The second rule says that if $T$ is an $H$-closed filter in $\Phi(Y)$, then so is $s_{*} T$ in $\Phi(X)$.

Now we can prove that if $H_{1}$ and $H_{2}$ are $L$-equivalent, then the categories $L K_{\Theta}\left(H_{1}\right)$ and $L K_{\Theta}\left(H_{2}\right)$ are correctly isomorphic.

Suppose that $H_{1}$ and $H_{2}$ are $L$-equivalent. We shall define an isomorphism $F$ : $L K_{\Theta}\left(H_{1}\right) \rightarrow L K_{\Theta}\left(H_{2}\right)$. Let $(X, A)$ be an object in $L K_{\Theta}\left(H_{1}\right)$. We set $F(X, A)=(X, B)$, where $B=\left(A_{H_{1}}^{L}\right)_{H_{2}}^{L}$. Here $F$ determines a bijection on the objects of the category.

Take a morphism $[s]=[s]_{H_{1}}:\left(X, A_{1}\right) \rightarrow\left(X, A_{2}\right)$ in $L K_{\Theta}\left(H_{1}\right)$. We have $s: W(Y) \rightarrow$ $W(X)$ and $\tilde{s}: \operatorname{Hom}\left(W(X), H_{1}\right) \rightarrow \operatorname{Hom}\left(W(Y), H_{1}\right)$. If $\nu \in A_{1}$, then $\tilde{s}(\nu) \in A_{2}$. We check that for the same $s$ we have $\tilde{s}(\mu) \in B_{2}$ if $\mu \in B_{1}$. Here $B_{1}=\left(A_{1 H_{1}}^{L}\right)_{H_{2}}^{L}, B_{2}=$ $\left(A_{2 H_{1}}^{L}\right)_{H_{2}}^{L}$. Our aim is to define $[s]_{H_{2}}:\left(X, B_{1}\right) \rightarrow\left(X, B_{2}\right)$. The embedding $\tilde{s}(\nu)=\nu s \in$ $A_{2}$ means that $\nu \in \tilde{s}^{-1} A_{2}=s_{*} A_{2}$. This is equivalent to $A_{1} \subset s_{*} A_{2}$. We have

$$
\left(A_{1 H_{1}}^{L}\right) \supset\left(s_{*} A_{2}\right)_{H_{1}}^{L} \supset s^{*} A_{2 H_{1}}^{L}
$$

and

$$
\left(A_{1 H_{1}}^{L}\right)_{H_{2}}^{L}=B_{1} \subset\left(s^{*} A_{2 H_{1}}^{L}\right)_{H_{2}}^{L}=s_{*}\left(A_{2 H_{1}}^{L}\right)_{H_{2}}^{L}=s_{*} B_{2} .
$$

Thus, $B_{1} \subset s_{*} B_{2}$, and we have $\tilde{s}(\mu) \in B_{2}$ for every $\mu \in B_{1}$. Similarly we can check that if $\tilde{s}(\mu) \in B_{2}$ for every $\mu \in B_{1}$, then $\tilde{s}(\nu) \in A_{2}$ for every $\nu \in A_{1}$.

We show that for $s_{1}, s_{2}: W(Y) \rightarrow W(X)$ the relation $\tilde{s}_{1}(\nu)=\tilde{s}_{2}(\nu)$ for every $\nu \in$ $A_{1}=A$ is equivalent to $\tilde{s}_{1}(\mu)=\tilde{s}_{2}(\mu)$ for every $\mu \in B_{1}=B$.

Let $\tilde{s}_{1}(\nu)=\nu s_{1}=\nu s_{2}=\tilde{s}_{2}(\nu)$ be given. For every $w \in W(Y)$ we have $\nu s_{1}(w)=$ $\nu s_{2}(w)$. Consider the equation $s_{1} w \equiv s_{2} w$. Then $\nu$ is a solution of this equation. This gives $A \subset \operatorname{Val}_{H_{1}}^{X}\left(s_{1} w \equiv s_{2} w\right)$, and $s_{1} w \equiv s_{2} w \in A_{H_{1}}^{L}$. Since $A_{H_{1}}^{L}=B_{H_{2}}^{L}$, we have $s_{1} w \equiv s_{2} w \in B_{H_{2}}^{L}$ and $B \subset \operatorname{Val}_{H_{2}}^{X}\left(s_{1} w \equiv s_{2} w\right)$. This means that for every $\mu \in B$ we have $\mu s_{1} w=\mu s_{2} w$. This is true for every $w \in W(Y)$, so that $\mu s_{1}=\mu s_{2}$, i.e., $\tilde{s}_{1}(\mu)=\tilde{s}_{2}(\mu)$. The converse statement is also true. Now it is clear that $F\left([s]_{H_{1}}\right)=[s]_{H_{2}}$ is well defined, because it does not depend on the choice of a representative. Thus, we get an isomorphism of categories: $F: L K_{\Theta}\left(H_{1}\right) \rightarrow L K_{\Theta}\left(H_{2}\right)$.

The lattice $\operatorname{Lat}_{H_{1}}(W)$ of all elementary sets in $\operatorname{Hom}\left(W(X), H_{1}\right)$ is anti-isomorphic to the lattice of $H_{1}$-closed filters in $\Phi(X)$. The latter is anti-isomorphic to the lattice $\operatorname{Lat}_{H_{2}}(W)$. Here $\operatorname{Lat}_{H_{1}}(W)$ and $\operatorname{Lat}_{H_{2}}(W)$ are isomorphic. The isomorphism is induced by the initial isomorphism of categories $F$. The theorem is proved.

It would be quite natural to expect that $F$ induces an isomorphism of functors $\mathrm{LCl}_{H_{1}} \rightarrow \mathrm{LCl}_{H_{2}}$. This would mean that for every $s: W(X) \rightarrow W(Y)$ and every elementary set $A \subset \operatorname{Hom}(W(X, H))$ we have $\left(s_{*}\left(A_{1 H_{1}}^{L}\right)\right)_{H_{2}}^{L}=s_{*} B, B=\left(A_{1 H_{1}}^{L}\right)_{H_{2}}^{L}$. But we can prove only the inclusion $B \subset\left(A_{1 H_{1}}^{L}\right)_{H_{2}}^{L}$.

3.4. Automorphic LG-equivalence of algebras. The notion of automorphic equivalence generalizes the notion of LG-equivalence of algebras. Consider the diagram

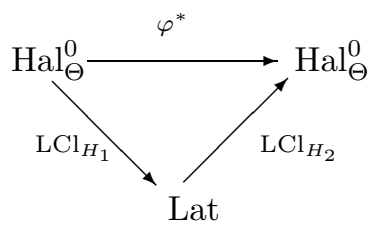

Here $\varphi^{*}$ is the automorphism of the category $\operatorname{Hal}_{\Theta}^{0}$ corresponding to $\varphi: \Theta^{0} \rightarrow \Theta^{0}$. The commutativity of this diagram means that there is a transition $\beta(\varphi): \mathrm{LCl}_{H_{1}} \rightarrow \mathrm{LCl}_{H_{2}} \varphi^{*}$ 
such that for every object $\Phi=\Phi(X)$ of $\operatorname{Hal}_{\Theta}^{0}$ there is an isomorphism of lattices

$$
\beta(\varphi)_{\Phi}: \mathrm{LCl}_{H_{1}}(\Phi) \rightarrow \mathrm{LCl}_{H_{2}} \varphi^{*}(\Phi) .
$$

Note that $\beta(\varphi)$ is not necessarily an isomorphism of functors. Next, assume that $\varphi$ and $\beta(\varphi)$ are coordinated in the following way.

1. Let $s: W(Y) \rightarrow W(X)$, and accordingly, $s_{*}: \Phi(Y) \rightarrow \Phi(X)$ be given. We also have $\tilde{s} \operatorname{Hom}\left(W(X), H_{1}\right) \rightarrow \operatorname{Hom}\left(W(Y), H_{1}\right)$. Take the elementary sets $A_{1} \subset \operatorname{Hom}\left(W(X), H_{1}\right)$ and $A_{2} \subset \operatorname{Hom}\left(W(Y), H_{1}\right)$, and put $B_{1}=\beta(\varphi)_{\Phi(X)}\left(A_{1}\right), B_{2}=\beta(\varphi)_{\Phi(Y)}\left(A_{2}\right)$. Assume that $\tilde{s}(\nu) \in A_{2}$ for $\nu \in A_{1}$ if and only if $\widetilde{\varphi(s)}(\mu) \in B_{2}$ for $\mu \in B_{1}$.

2. Let $s_{1}, s_{2}: W(Y) \rightarrow W(X)$ be given, and let $\tilde{s}_{1}, \tilde{s}_{2}: \operatorname{Hom}\left(W(X), H_{1}\right) \rightarrow$ $\operatorname{Hom}\left(W(Y), H_{1}\right)$. Take an elementary set $A \subset \operatorname{Hom}\left(W(X), H_{1}\right)$ and put $B=\beta(\varphi)_{\Phi(X)}(A)$. Assume that $\tilde{s}_{1}(\nu)=\tilde{s}_{2}(\nu)$ for every $\nu \in A$ if and only if $\widetilde{\varphi\left(s_{1}\right)}(\mu)=\widetilde{\varphi\left(s_{2}\right)}(\mu)$ for every $\mu \in B$.

These commutativity conditions determine the notion of automorphic LG-equivalence for the algebras $H_{1}$ and $H_{2}$. LG-equivalence is a particular case of automorphic LGequivalence; this case arises when $\varphi=1$ is the identity automorphism and $\beta(1)_{\Phi}(A)=$ $\left(A_{H_{1}}^{L}\right)_{H_{2}}^{L}$. The conditions for $\varphi=1$ and $\beta(1)$ were checked in the proof of Theorem 1.14. The next theorem is proved in a similar way.

Theorem 3.1. If $H_{1}$ and $H_{2}$ are automorphically $L G$-equivalent, then the categories $L K_{\Theta}\left(H_{1}\right)$ and $L K_{\Theta}\left(H_{2}\right)$ are correctly isomorphic.

Remark 3.2. Observe that, in the case of algebraic geometry, if $\varphi$ is inner, then the corresponding $H_{1}$ and $H_{2}$ are AG-equivalent. In the situation of logical geometry we cannot prove a similar statement. We also cannot prove that $\beta(\varphi)$ is an isomorphism of functors. In the situation of AG this is always the case.

3.5. Coordinate algebras. The category $L C_{\Theta}(H)$. Let $A$ be an elementary set in $\operatorname{Hom}(W(X), H)$. Consider $T=A_{H}^{L}$. The algebra $\Phi(X) / T$ is the coordinate Boolean algebra for the set $A$.

Given a formula $u \in \Phi(X)$, we denote by $\tilde{u}$ the characteristic Boolean function of the set $\left(\operatorname{Val}_{H}^{X}\right)(u)$ in $\operatorname{Hom}(W(X), H)$ and by $\tilde{u}_{A}$ the specialization of the function $\tilde{u}$ to the set $A$. It is known [P6] that $\Phi(X) / T$ is isomorphic to the algebra of all such $\tilde{u}_{A}$.

We define the category $L C_{\Theta}(H)$ of all $\Phi(X) / T$. As usual, consider $s: W(Y) \rightarrow W(X)$ and $s_{*}: \Phi(Y) \rightarrow \Phi(X)$. Consider the corresponding filters $T_{2}$ in $\Phi(Y)$ and $T_{1}$ in $\Phi(X)$. We say that $s$ is admissible with respect to $T_{1}$ and $T_{2}$ if $s_{*} u \in T_{1}$ for every $u \in T_{2}$. In this case we have a homomorphism $\bar{s}=\bar{s} *: \Phi(Y) / T_{2} \rightarrow \Phi(X) / T_{1}$. All these $\bar{s}$ for $H$-closed $T_{1}$ and $T_{2}$ are taken as morphisms in the category $L C_{\Theta}(H)$. Let $A_{1}$ and $A_{2}$ be elementary sets, and let $T_{1}=A_{1 H}^{L}, T_{2}=A_{2 H}^{L}$. We say that $s: W(Y) \rightarrow W(X)$ is admissible with respect to $A_{1}$ and $A_{2}$ if $\tilde{s}(\nu) \in A_{2}$ for $\nu \in A_{1}$.

Proposition 3.3 ([י] $]$ ). The homomorphism $s: W(Y) \rightarrow W(X)$ is admissible for elementary sets $A_{1}$ and $A_{2}$ if and only if $s$ is admissible for $T_{2}$ and $T_{1}$.

Next, suppose that $\left[s_{1}\right]$ and $\left[s_{2}\right]$ coincide as morphisms in $\left(X, A_{1}\right) \rightarrow\left(Y, A_{2}\right)$. Then $\bar{s}_{1}$ and $\bar{s}_{2}$ coincide as morphisms in $\Phi(Y) / T_{2} \rightarrow \Phi(X) / T_{1}$ (see [P6]). This gives a contravariant functor $L K_{\Theta}(H) \rightarrow L C_{\Theta}(H)$.

However, we cannot state that $\bar{s}_{1}=\bar{s}_{2}$ implies $\left[s_{1}\right]=\left[s_{2}\right]$ because of the lack of duality. Duality appears if the following separability condition is fulfilled: if $s_{1} \neq s_{2}$ and for some point $\nu$ we have $\nu s_{1} \neq \nu s_{2}$, then $\operatorname{Val}_{H}(u)$ contains one of these points and does not contain the other for some $u$. This type of separability occurs if the algebra $H$ consists of constants. 
The next proposition demonstrates the role of constants in this setting. Now we consider varieties of the type $\Theta^{G}$. Here $G \in \Theta$ is the algebra of constants. Each algebra $H \in \Theta^{G}$ admits an embedding $h: G \rightarrow H$, not necessarily faithful. The morphisms are of the form

The free algebras in $\Theta^{G}$ have the form

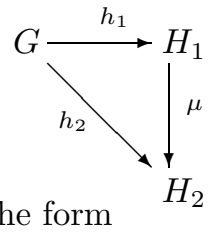

$$
i_{G}: G \rightarrow G * W_{0}(X)=W(X) .
$$

Here $W_{0}(X)$ is a free algebra in $\Theta, *$ is the sign of the free product in $\Theta, i_{G}$ is the embedding in the definition of the free product. The algebra $G$ is an algebra in $\Theta^{G}$ with the identical morphism $\epsilon: G \rightarrow G$. The diagram

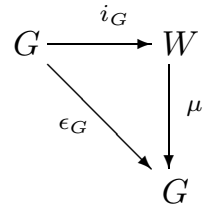

implies that for every $g \in G$ and every $\mu$ we have $\mu(g)=g$. Moreover, $s(g)=g$ for every $g \in G$ and every $s: W(Y) \rightarrow W(X)$ in $\Theta^{G}$.

Now we take the algebra $G$ as an algebra where we are looking for solutions of equations, i.e., $G$ plays the role of $H$. Then we have the following statement.

Proposition 3.4. The equality $\bar{s}_{1}=\bar{s}_{2}$ implies the equality $\left[s_{1}\right]=\left[s_{2}\right]$.

Proof. The equality $\left[s_{1}\right]=\left[s_{2}\right]$ means that every point $\nu \in A$ satisfies every equation $s_{1} w \equiv s_{2} w, w \in W(Y)$. Consider the equality $\bar{s}_{1}=\bar{s}_{2}: \Phi(Y) / T_{2} \rightarrow \Phi(X) / T_{1}$. This means that for every $u \in \Phi(Y)$, and accordingly for every $\bar{u} \in \Phi(Y) / T_{2}$, we have $\bar{s}_{1}(\bar{u})=$ $\bar{s}_{2}(\bar{u}) ; \overline{s_{1 *}(u)}=\overline{s_{2 *}(u)}$. This is an equality in $\Phi(X) / T_{1}$ and it means that a formula of the form

$$
v=\left(s_{1 *}(u) \rightarrow s_{2 *}(u)\right) \wedge\left(s_{2 *}(u) \rightarrow s_{1 *}(u)\right)
$$

is contained in the filter $T_{1}=A_{G}^{L}$. We have $A \subset \operatorname{Val}_{G}(v)$. Denote $\operatorname{Val}_{G}(u)=C$. Then $\operatorname{Val}_{G}(v)=\left(s_{1 *} C \rightarrow s_{2 *} C\right) \wedge\left(s_{2 *} C \rightarrow s_{1 *} C\right)$.

We fix $w \in W(Y)$ and $\nu \in A$. Let $\nu s_{1}(w)=g \in G$. Consider the equation $w \equiv g$. We take this equation as $u \in \Phi(Y)$ and let $C=\operatorname{Val}_{G}(w \equiv g)$. Here $s_{1 *} w \equiv g$. The point $\nu$ satisfies this equation, i.e., $\nu \in s_{1 *} C$. Then $\nu \in s_{2 *} C, \nu s_{2} w \equiv g$. Hence, $\nu s_{1} w=\nu s_{2} w=g$.

On the other hand, one can proceed from $\nu s_{2} w=g_{1}$ for the same $\nu$ and $w$. Let us look again at $w \equiv g_{1}$. Using the formula for $\operatorname{Val}_{G}(v)$, we obtain $\nu s_{2} w=\nu s_{1} w \equiv g_{1}$. Since $\nu$ belongs to both parts of the formula for $\operatorname{Val}_{G}(v)$, we get $g=g_{1}$. In both cases $\nu s_{1}(w)=\nu s_{2}(w)$ for all $\nu$ and $w$, and the proposition is proved. So, we come up with the duality $L K_{\Theta^{G}}(G) \rightarrow L C_{\Theta^{G}}(G)$.

3.6. More about automorphic equivalence of algebras. Consider the diagram

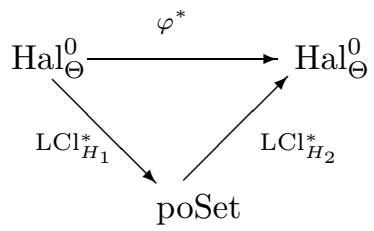


We describe the meaning of the commutativity of this diagram. Consider a transition (not necessarily an isomorphism) $\alpha(\varphi): L C l_{H_{1}}^{*} \rightarrow L C l_{H_{2}}^{*} \varphi^{*}$. Suppose that for every object $\Phi=\Phi(X)=\operatorname{Hal}_{\Theta}^{0}(X)$ there is an isomorphism of $H$-closed filters:

$$
\alpha(\varphi): L C l_{\Phi}^{*} \rightarrow L C l_{H_{2}}^{*} \varphi^{*} .
$$

Suppose also that $\alpha(\varphi)$ and $\varphi$ are coordinated in the following sense.

1. Let $s: W(Y) \rightarrow W(X)$, and accordingly, $s_{*}: \Phi(Y) \rightarrow \Phi(X)$, be given. Consider the corresponding filters $T_{2}$ in $\Phi_{2}=\Phi(Y)$ and $T_{1}$ in $\Phi_{1}=\Phi(X)$. Take $\alpha(\varphi)_{\Phi_{1}}\left(T_{1}\right)=T_{1}^{*}$ and $\alpha(\varphi)_{\Phi_{2}}\left(T_{2}\right)=T_{2}^{*}$. The coordination condition says that $s_{*}$ is admissible with respect to $T_{2}$ and $T_{1}$ if and only if $\varphi^{*}\left(s_{*}\right)=\varphi(s)_{*}$ is admissible with respect to $T_{2}^{*}$ and $T_{1}^{*}$.

2. Let $s_{1}, s_{2}: W_{2} \rightarrow W_{1}$ be given, and let $s_{1 *}, s_{2 *}: \Phi_{2} \rightarrow \Phi_{1}$. Let $T_{1}$ be an $H_{1}$-closed filter in $\Phi_{1}$, let $\mu: \Phi_{1} \rightarrow \Phi_{1} / T_{1}$ be the natural homomorphism, and let $\alpha(\varphi)_{\Phi_{1}}\left(T_{1}\right)=T_{1}^{*}$ and $\mu^{*}: \varphi^{*}\left(\Phi_{1}\right) \rightarrow \varphi^{*}\left(\Phi_{1}\right) / T_{1}^{*}$. The coordination says that $\mu s_{1 *}=\mu s_{2 *}$ if and only if $\mu^{*} \varphi\left(s_{1}\right)_{*}=\mu^{*} \varphi\left(s_{2}\right)_{*}$.

The diagram above is commutative if it satisfies conditions 1 and 2 . Now we relate $\alpha(\varphi)$ and $\beta(\varphi)$. Suppose that

$$
\begin{aligned}
& \alpha(\varphi)_{\Phi}(T)=\beta(\varphi)_{\Phi}\left(T_{H_{1}}^{L}\right)_{H_{2}}^{L}, \\
& \beta(\varphi)_{\Phi}(A)=\alpha(\varphi)_{\Phi}\left(A_{H_{1}}^{L}\right)_{H_{2}}^{L} .
\end{aligned}
$$

Under these conditions, we have the following statement.

Proposition 3.5. $\alpha(\varphi)$ and $\varphi$ are coordinated if and only if $\beta(\varphi)$ and $\varphi$ are coordinated.

This is proved by a straightforward check.

Now we can say that the algebras $H_{1}$ and $H_{2}$ are automorphically LG-equivalent if and only if there is a commutative diagram

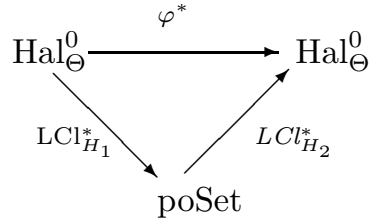

It can be proved that if $H_{1}$ and $H_{2}$ are $L G$-equivalent, then there is a correct isomorphism of categories $L C_{\Theta}\left(H_{1}\right) \rightarrow L C\left(H_{2}\right)$.

Note that in the situation of algebraic geometry all the coordination conditions above allow us to compute $\alpha(\varphi)$ and $\beta(\varphi)$ explicitly. Moreover, these transitions turn out to be isomorphisms of functors. In logical geometry this is not the case.

3.7. LG-similarity of algebras. Our main goal is to study necessary and sufficient conditions for the algebras $H_{1}$ and $H_{2}$ to have the same logic. First, observe that if $H$ is an algebra in the variety $\Theta$, and $\Theta_{1}$ is a subvariety in $\Theta$ containing $H$, then the categories $L K_{\Theta}(H)$ and $L K_{\Theta_{1}}(H)$ are canonically isomorphic. This follows from the observations in Subsection 2.8.

Denote $\Theta_{1}=\operatorname{Var}\left(H_{1}\right)$ and $\Theta_{2}=\operatorname{Var}\left(H_{2}\right)$. Now we are able to relate the categories $L K_{\Theta_{1}}\left(H_{1}\right)$ and $L K_{\Theta_{2}}\left(H_{2}\right)$. Consider the diagrams

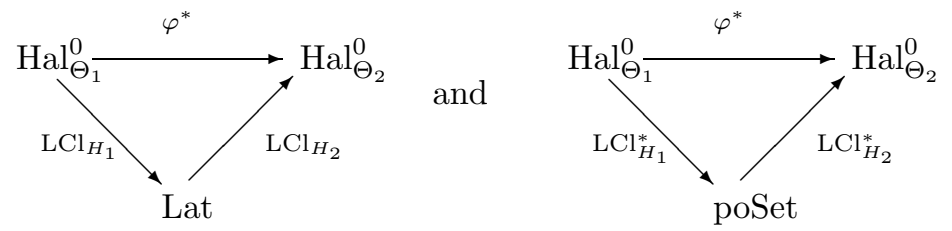

The commutativity of these diagrams is defined in accordance with the scheme used in the definition of automorphic LG-equivalence of algebras. Here $\alpha(\varphi): \mathrm{LCl}_{H_{1}}^{*} \rightarrow \mathrm{LCl}_{H_{2}}^{*} \varphi^{*}$ 
and $\beta(\varphi): \mathrm{LCl}_{H_{1}}^{*} \rightarrow \mathrm{LCl}_{H_{2}}^{*} \varphi^{*}$ are such that for every $\Phi=\Phi(X)=\operatorname{Hal}_{\Theta_{1}}^{0}(X)$ we have an isomorphism of lattices

$$
\begin{aligned}
\alpha(\varphi)_{\Phi}: L C l_{H_{1}}^{*}(\Phi) \rightarrow L C l_{H_{2}}^{*}\left(\varphi^{*}(\Phi)\right), \\
\beta(\varphi)_{\Phi}: L C l_{H_{1}}(\Phi) \rightarrow L C l_{H_{2}}\left(\varphi^{*}(\Phi)\right) .
\end{aligned}
$$

Here, $\alpha(\varphi), \beta(\varphi)$, and $\varphi$ are related by the rules cited above. This implies that the diagrams are commutative simultaneously.

Definition 3.6. The algebras $H_{1}$ and $H_{2}$ are said to be logically similar if there exists $\varphi: \Theta^{0} \rightarrow \Theta^{0}$ such that the diagrams above are commutative.

Theorem 3.7. The following conditions are equivalent:

1) $H_{1}$ and $H_{2}$ are logically similar;

2) the categories $L K_{\Theta_{1}}\left(H_{1}\right)$ and $L K_{\Theta_{2}}\left(H_{2}\right)$ are correctly isomorphic;

3) the categories $L C_{\Theta_{1}}\left(H_{1}\right)$ and $L C_{\Theta_{2}}\left(H_{2}\right)$ are correctly isomorphic.

The proof is similar to the arguments used in [P4].

Now some remarks about the isomorphism $\varphi: \Theta_{1}^{0} \rightarrow \Theta_{2}^{0}$ are in order. Consider the category $L C_{\Theta}(H)$. Its objects are of the form $\operatorname{Hal}_{\Theta}^{0}(X) / T=\Phi(X) / T$, where $T$ is an $H$-closed filter in $\Phi$. Consider its full subcategory defined by objects $\operatorname{Hal}_{\Theta}^{0}(X) / T h^{X}(H)$. Recall that the elementary theory $T h^{X}(H)$ is the minimal $H$-closed filter in $\Phi(X)$. Moreover, if $u \in T h^{X}(H)$ and $s_{x}: \Phi(X) \rightarrow \Phi(Y)$ is a morphism in $\operatorname{Hal}_{\Theta}^{0}$, then $s_{*} u \in T h^{X}(H)$. We denote the resulting subcategory by Hal ${ }_{\Theta}^{\diamond}$. Let an isomorphism $Q: L C_{\Theta_{1}}\left(H_{1}\right) \rightarrow L C_{\Theta_{2}}\left(H_{2}\right)$ be given. Suppose that $Q$ is correct, which means that it induces an isomorphism $\psi: \mathrm{Hal}_{\Theta_{1}}^{\diamond} \rightarrow \mathrm{Hal}_{\Theta_{2}}^{\diamond}$. Assume also that an isomorphism $\varphi: \Theta_{1}^{0} \rightarrow \Theta_{2}^{0}$ induces $\varphi^{*}: \mathrm{Hal}_{\Theta_{1}}^{0} \rightarrow \mathrm{Hal}_{\Theta_{2}}^{0}$, and $\varphi^{*}$ induces $\psi$. The correctness condition for $Q$ is parallel to the condition that an isomorphism $F: L K_{\Theta_{1}}\left(H_{1}\right) \rightarrow L K_{\Theta_{2}}\left(H_{2}\right)$ induces an isomorphism of affine spaces $K_{\Theta_{1}}^{0}\left(H_{1}\right) \rightarrow K_{\Theta_{2}}^{0}\left(H_{2}\right)$.

We conclude this subsection with some general remarks. We compare the situations of $A G$ and $L G$.

In AG, an investigation of automorphisms $\varphi: \Theta^{0} \rightarrow \Theta^{0}$ or isomorphisms $\varphi: \Theta_{1}^{0} \rightarrow \Theta_{2}^{0}$ leads to a description of constructive algebraic relations between the algebras $H_{1}$ and $H_{2}$ with the same algebraic geometry. In LG, there are no general rules to find out whether the logics are the same. The problem should be considered at the level of the particular algebras $H_{1}$ and $H_{2}$. In AG, automorphic equivalence with an inner automorphism $\varphi$ implies AG-equivalence. There is no reduction of this sort in LG. In AG the transitions $\alpha(\varphi)$ and $\beta(\varphi)$ are isomorphisms of functors determined by explicit formulas. In LG this is impossible. All these properties determine mainstreams of investigations. In logical geometry, the main problems are related to ideas of LG-equivalence and the property of being LG-Noetherian. Such an LG-theory is considered with respect to different varieties $\Theta$ and specific $H \in \Theta$. Here there is much individuality for each particular case. One can expect interesting results for the variety of Abelian groups, the variety of modules over a ring $K$, or in the case where $\Theta$ is the variety of all commutative, associative algebras over a field $\Theta=C o m-P$.

We make some observations about logically Noetherian algebras. All necessary definitions can be taken from $\S 1$. Similar definitions work also in AG.

Note that if $H$ is an LG-Noetherian algebra, then $H$ is not necessarily AG-Noetherian. Indeed, if $T$ is a system of equalities in $\Phi(X)$, and $H$ is LG-Noetherian, then $T_{H}^{L}=$ $T_{H}^{\prime}=\operatorname{Val}_{H}^{X}(u)$. However, the formula $u$ may fail to be an equality. Therefore, $T$ is not necessarily reduced to one equality. On the other hand, if an algebra $H$ is strictly or weakly LG-Noetherian, then it is strictly or weakly AG-Noetherian. 
Now we show that if $H_{1}$ and $H_{2}$ are weakly LG-Noetherian, then their elementary equivalence implies their LG-equivalence. Indeed, suppose $T \rightarrow v$ holds in $H_{1}$. We take a finite $T_{0} \subset T$ such that $T_{0} \rightarrow v$ holds in $H_{1}$. Elementary equivalence implies that $T_{0} \rightarrow v$ holds in $H_{2}$. Hence, $T \rightarrow v$ holds in $H_{2}$.

In the general case, elementary equivalence does not imply LG-equivalence. Indeed, take $H=H_{1}$ that is not weakly AG-Noetherian. There is an ultrapower $H_{2}$ of $H$ such that $H_{1}$ and $H_{2}$ are not AG-equivalent (see [MR1, P4]). Therefore, $H_{1}$ and $H_{2}$ are not LG-equivalent. However, they are elementary equivalent.

There are also specific examples of elementary equivalent algebras that are not LGequivalent. For instance, if $L_{1}$ and $L_{2}$ are two extensions of a ground field $P$, then they can be elementary equivalent, but not LG-equivalent. In the case of $\Theta=C o m-P$ we have the AG-Noetherian property (Hilbert's basis theorem), but not the LG-Noetherian property.

Group theory provides other examples. Two free groups $F_{n}$ and $F_{m}$ with different $n \geq 2$ and $m \geq 2$ are elementary equivalent but not LG-equivalent.

It is easily seen that every finite algebra is LG-Noetherian. However, no infinite algebras with such a property have yet been constructed.

3.8. Elementary sets over $H$ and the group $\operatorname{Aut}(H)$. The group of automorphisms $\operatorname{Aut}(H)$ acts in $\operatorname{Hom}(W, H)$. If $\mu: W \rightarrow H$ is a point and $g \in \operatorname{Aut}(H)$, then $g \mu(w)=$ $g(\mu(w))$.

Theorem 3.8 (see [P6]). Every elementary set $A \subset \operatorname{Hom}(W, H)$ is invariant with respect to the action of Aut $H$.

If $H$ is a finite algebra, then the converse statement is also true: if a set $A \subset$ $\operatorname{Hom}(W, H)$ is invariant with respect to the action of Aut $H$, then $A$ is an elementary set.

It can be proved that $\operatorname{Aut}(H)$ is naturally isomorphic to the automorphism group of the algebra $\mathrm{Hal}_{\Theta}(H)$. For finite $H$ this leads to a Galois correspondence that relates subgroups in $\operatorname{Aut}(H)$ and subalgebras in $\operatorname{Hal}_{\Theta}(H)$.

3.9. Results for specific varieties. It is easy to prove that two free (finitely generated) semigroups are isomorphic if they are elementary equivalent. The same statement is true for free inverse semigroups. On the other hand, if two semigroups (or inverse semigroups) are elementary equivalent and one of them is free, then the other semigroup may fail to be free. The following results show that LG-equivalence is stronger in this case.

Theorem 3.9. Every free semigroup is separable in the variety of all semigroups.

Proof. Let $S=S(X)$ be a free semigroup over a set $X=\left\{x_{1}, \ldots, x_{n}\right\}$ of free generators. Let $H$ be a semigroup logically equivalent to $S$. Consider the following formulas in the semigroup language:

$$
\begin{array}{ll}
\text { A1 : } & \bigwedge_{i \neq j, i, j=1, \ldots n}\left(x_{i} \not \equiv x_{j}\right), \\
\text { A2 : } & \left(\forall x_{n+1} \not \equiv x_{1}, \ldots, x_{n}\right)\left(\exists ! x_{n+2}\right)\left(x_{n+1} \equiv x_{1} x_{n+2} \vee \cdots \vee x_{n+1} \equiv x_{n} x_{n+2}\right), \\
\text { A3 : } & \bigwedge_{i, j=1, \ldots n}\left(\forall x_{n+1}\right)\left(x_{i} \not \equiv x_{j} x_{n+1}\right), \\
\text { A4 : } & \left(\forall x_{1}\right)\left(\forall x_{2}\right)\left(\forall x_{3}\right)\left(x_{1} x_{3} \equiv x_{2} x_{3} \Rightarrow x_{1} \equiv x_{2}\right),
\end{array}
$$

and the following family of formulas:

$$
B_{i_{1}, i_{2}, \ldots, i_{k}}: \quad x_{n+1} \not \equiv x_{i_{1}} x_{i_{2}} \cdots x_{i_{k}},
$$

where $i_{1}, i_{2}, \ldots, i_{k} \in\{1,2, \ldots, n\}, k \in \mathbb{N}$. 
The meaning of these formulas is obvious. Now we construct the conjunction of the first four formulas and close the resulting formula in the following way:

$$
\left(\exists x_{1}, \ldots, x_{n}\right)(A 1 \wedge A 2 \wedge A 3) \wedge A 4 .
$$

If the elementary formula (3.1) is true in a semigroup $H$, then there exist $n$ pairwise different elements $a_{1}, \ldots, a_{n}$ in $H$ such that every element $a$ different from these $n$ elements can be represented as $a=a_{i} b$ for some $i=1, \ldots, n$ and some element $b \in H$, and this representation is unique.

Obviously, the sentence (3.1) is true in $S$, and the only solutions of the formula $A 1 \wedge A 2 \wedge A 3$ are $n$-tuples of pairwise different elements $x_{1}, \ldots, x_{n}$ ordered in some way. Thus, (3.1) is true in $H$. Fix an $n$-tuple $\left(a_{1}, \ldots, a_{n}\right)$ of elements of $H$ that give a solution of the formula $A 1 \wedge A 2 \wedge A 3$.

Consider the infinite set $T$ of formulas $A 1, A 2, A 3, B_{i_{1}, i_{2}, \ldots, i_{k}}$. This set is an inconsistent system in $S$, because for all values of the variables $x_{1}, \ldots, x_{n}, x_{n+1}$, if the formulas $A 1, A 2, A 3$ are satisfied, then one of the formulas $B_{i_{1}, i_{2}, \ldots, i_{k}}$ is wrong. Thus, the implication

$$
T \rightarrow x_{1} \not \equiv x_{1}
$$

is true in $S$.

Consequently, this implication is true in $H$. Let $a$ be an arbitrary element of $H$. Let the free variables $x_{1}, \ldots, x_{n}, x_{n+1}$ have the following values in $H: a_{1}, \ldots, a_{n}, a$, respectively. Then one of the formulas in $\Gamma$ must be wrong. However, $A 1, A 2$, and $A 3$ are true. This means that one of the formulas $B_{i_{1}, i_{2}, \ldots, i_{k}}$ is wrong, whence $a$ is a composition of elements $a_{1}, \ldots, a_{n}$. It has been mentioned above that such a representation is unique.

Thus, $H$ is the free semigroup over the free generators $a_{1}, \ldots, a_{n}$, so that $H$ is isomorphic to $S$.

The case of inverse semigroups is more complicated, but the same idea is realized, that is, the free generators can be determined in terms of the first-order language.

We consider inverse semigroups as algebras with two operations, a binary multiplication $\cdot$ and a unary inversion ${ }^{-1}$ (here $a^{-1}$ is the inverse of an element $a$ ). The class of all inverse semigroups forms a variety defined by the identities

$$
\begin{gathered}
(x y) z \equiv x(y z), \quad(x y)^{-1} \equiv y^{-1} x^{-1}, \quad\left(x^{-1}\right)^{-1} \equiv x, \\
x x^{-1} x \equiv x, \quad x^{-1} x y^{-1} y \equiv y^{-1} y x^{-1} x
\end{gathered}
$$

Theorem 3.10. Every free inverse semigroup is separable in the variety of all inverse semigroups.

Proof. Let $S=S(X)$ be a free inverse semigroup over a set $X=\left\{x_{1}, \ldots, x_{n}\right\}$ of free generators. Let $H$ be an inverse semigroup logically equivalent to $S$. Consider the following formulas in the inverse semigroup language:

A1 :

$$
\bigwedge_{i \neq j, i, j=1, \ldots n}\left(x_{i} \neq x_{j}\right) \wedge \bigwedge_{i, j=1, \ldots n}\left(x_{i} \neq x_{j}^{-1}\right)
$$

A2 : $\quad\left(\forall x_{n+1} \neq x_{1}^{ \pm 1}, \ldots, x_{n}^{ \pm 1}\right)\left(\exists x_{n+2}\right)\left(x_{n+1}=x_{1}^{ \pm 1} x_{n+2} \vee \cdots \vee x_{n+1}=x_{n}^{ \pm 1} x_{n+2}\right)$,

A3 :

$$
\bigwedge_{i \neq j, i, j=1, \ldots n}\left(\forall x_{n+1}\right)\left(\left(x_{i} \not \equiv x_{j} x_{n+1}\right) \wedge\left(x_{i} \not \equiv x_{j}^{-1} x_{n+1}\right)\right) .
$$

Now we close the conjunction of these three formulas in the following way:

$$
\left(\exists x_{1}, \ldots, x_{n}\right)(A 1 \wedge A 2 \wedge A 3) .
$$

If the elementary formula (3.2) is true in an inverse semigroup $H$, then there exist $n$ pairwise different elements $a_{1}, \ldots, a_{n}$ in $H$ such that every element $a$ different from these 
$n$ elements can be represented either in the form $a=a_{i} b$, or in the form $a=a_{i}^{-1} b$ for some $i=1, \ldots, n$, and some element $b \in H$, and moreover, no element $a_{k}$ can be represented in this form if $k \neq i$.

Obviously, the sentence (3.2) is true in $S$, and the only solutions of the formula $A 1 \wedge A 2 \wedge A 3$ are $n$-tuples of elements $u_{1}, \ldots, u_{n}$ of the set $\left\{x_{1}, \ldots, x_{n}, x_{1}^{-1}, \ldots, x_{n}^{-1}\right\}$, where every $u_{i}$ is different from $u_{j}$ if $i \neq j$ and from $u_{j}^{-1}$. Thus, $u_{1}, \ldots, u_{n}$ are free generators of $S$. Since (3.2) is true in $S$, (3.2) is true in $H$.

Now, to every two different elements $w_{1}$ and $w_{2}$ of $S$, we assign the formula $w_{1} \not \equiv w_{2}$. To the resulting set of formulas we add the formulas $A_{1}, A_{2}, A_{3}$ and come up with a set $T_{1}$. It is obvious that $T_{1}$ is consistent in $S$. Therefore, $T_{1}$ has a solution in $H$. Fix an $n$-tuple $\left(a_{1}, \ldots, a_{n}\right)$ of elements of $H$ that gives a solution of all formulas in $T_{1}$.

Now, to every element $w$ of $S$ we assign the formula $x_{n+1} \not \equiv w$. Adding all these formulas to $T_{1}$, we obtain a set $T_{2}$. Obviously, $T_{2}$ is inconsistent in $S$. Thus, $T_{2}$ is inconsistent also in $H$. The function that takes every element $x_{i}$ to $a_{i}$ determines a homomorphism $\varphi: S \rightarrow H$. Since all formulas in $T_{1}$ are satisfied, this homomorphism is injective. Since one of the formulas $x_{n+1} \not \equiv w$ must be wrong, every element of $H$ belongs to the image of $S$, i.e., $\varphi: S \rightarrow H$ is surjective. Thus, $S$ and $H$ are isomorphic.

Applying the method used above, we can obtain a general and helpful fact.

Theorem 3.11. If two algebras $H_{1}$ and $H_{2}$ in the same variety are logically equivalent, then they contain the same free subalgebras, which means the following: if one of these algebras has a free subalgebra of range $n$, then the other algebra has a free subalgebra of the same range. Moreover, if $H_{1}$ contains a free proper subalgebra $F$, then $\mathrm{H}_{2}$ contains a proper subalgebra isomorphic to $F$.

Proof. Suppose $H_{1}$ has a free subalgebra $F$ of range $n$ with free generators $a_{1}, \ldots, a_{n}$. Consider the free algebra $W=W\left(x_{1}, \ldots, x_{n}\right)$ over $X=\left\{x_{1}, \ldots, x_{n}\right\}$ in our variety. For every two different elements $w_{1}$ and $w_{2}$ of $W$, we construct the formula $w_{1} \not \equiv w_{2}$. Let $T$ be the set of all such formulas. The set $T$ has a solution $a_{1}, \ldots, a_{n}$ in $H_{1}$. Then $T$ has a solution $b_{1}, \ldots, b_{n}$ in $H_{2}$.

Let $\eta: W \rightarrow B$ be a homomorphism that assigns the element $b_{i}$ to every $x_{i}$. Since all formulas in $T$ become true, $\eta$ is an injection of $W$ into $H_{2}$. Thus, $H_{2}$ has a subalgebra isomorphic to $F$.

Let $F$ be a proper free subalgebra of $H_{1}$. To the set $T$, we add all formulas of the form $x_{n+1} \not \equiv w$, where $w \in W$. The resulting set $T_{1}$ is consistent in $H_{1}$, whence $T_{1}$ is consistent in $H_{2}$. The solution of $T_{1}$ in $H_{2}$ generates a proper subalgebra isomorphic to $F$.

Corollary 3.12. If there is no proper subalgebra of a free algebra $F$ isomorphic to $F$, then $F$ is separable in the corresponding variety.

Proof. Let $H$ be logically equivalent to $F$. Then $H$ has a subalgebra isomorphic to $F$. This subalgebra cannot be a proper subalgebra of $H$ if $F$ has no proper free subalgebras isomorphic to $F$.

It is known that there are varieties with free algebras satisfying the condition mentioned in the corollary above, for example, the variety generated by a finite group.

It is known that elementary theories pass through any ultrafilter. Below we show that this is not the case for implicative theories.

We recall $[\mathrm{CK}]$ that a filter $\mathfrak{F}$ on an infinite set $I$ is said to be countably complete if the intersection of every countable set of elements of $\mathfrak{F}$ is an element of $\mathfrak{F}$. Otherwise, this filter is countably incomplete. 
Let $F$ be a countable free algebra. Obviously, if an ultrafilter $\mathfrak{F}$ is countably complete, then the corresponding ultrapower $H=F^{\mathfrak{F}}$ is logically equivalent to $F$. But the existence of such ultrafilters is problematic, and moreover, $H=F^{\mathfrak{F}}$ is isomorphic to $H$. Thus, this kind of ultrapower is not interesting.

Theorem 3.13. Let $F$ be a free algebra satisfying some nontrivial implicative formula $T \rightarrow v$ with countable $T$. If $\mathfrak{F}$ is a countably incomplete ultrafilter, then the corresponding ultrapower $G=F^{\mathfrak{F}}$ is not logically equivalent to $F$.

Proof. Since $\mathfrak{F}$ is countably incomplete, there exists a countable collection $A_{1}, \ldots, A_{n}, \ldots$ of subsets of $I$ such that they all belong to $\mathfrak{F}$ but the intersection of them is empty. Obviously, we may assume that $A_{1} \supset \cdots \supset A_{n} \supset \cdots$.

Consider a set $T$ of formulas constructed on the base of $n$ variables and such that $T$ is in contradiction (has no solution in $F$ ), but every finite subset of $T$ is consistent (has a solution in $F$ ). Let $T=\left\{\gamma_{1}, \gamma_{2}, \ldots\right\}$. Instead of these formulas, we can take the formulas $\gamma_{1}, \gamma_{1} \wedge \gamma_{2}, \ldots, \gamma_{1} \wedge \gamma_{2} \wedge \cdots \wedge \gamma_{m}, \ldots$. Therefore, we may assume from the very outset that $\gamma_{k} \Rightarrow \gamma_{k-1}$ is true for all $k=2,3, \ldots$. Let $\vec{u}_{k}=\left(u_{1 k}, \ldots, u_{n k}\right)$ denote a solution of $\gamma_{k}$, that is, the sentence $\gamma_{k}\left(\vec{u}_{k}\right)$ is true. Then all sentences $\gamma_{k}\left(\vec{u}_{m}\right)$ are true for $m<k$.

Let $i \in A_{1}$. Since the intersection of all sets $A_{k}$ is empty, there exists $m$ such that $i \notin A_{m}$. We construct an element of $H=F^{\mathfrak{F}}$ with the help of the following function: $f(i)=\vec{u}_{k}$, where $k$ is the greatest number such that $i \in A_{k}$, that is, $i \notin A_{k+1}$ and $f(i)=\vec{u}_{1}$ if $i \notin A_{1}$.

Now, let $i \in A_{m}$. There exists $k$ such that $i \in A_{k} \backslash A_{k+1}$. Thus, $f(i)=\vec{u}_{k}$ and $\gamma_{k}\left(\vec{u}_{k}\right)$ is true. Since $k \geq m$, we see that $\gamma_{m}(f(i))=\gamma_{m}\left(\vec{u}_{k}\right)$ is also true. Hence, the set $A$ of all elements $i \in I$ such that $\gamma_{m}(f(i))$ is true includes $A_{m}$, and therefore, this set $A$ belongs to the ultrafilter $\mathfrak{F}$. We have proved that the function $f$ determines a solution of all formulas in $T$.

\section{REFERENCES}

[CK] C. C. Chang and H. J. Keisler, Model theory, Stud. Logic Found. Math., vol. 73, North-Holland Publ. Co., Inc., New York, 1973. MR0409165 (53:12927)

[G] R. Grossberg, Classification theory for abstract elementary classes, Logic and Algebra (Yi Zhang, ed.), Contemp. Math., vol. 302, Amer. Math. Soc., Providence, RI, 2002, pp. 165-204. MR.1928390(2003h:03053)

[H] P. R. Halmos, Algebraic logic, Chelsea Publ. Co., New York, 1962. MR0131961 (24:A1808)

[HMT] L. Henkin, J. D. Monk, and A. Tarski, Cylindric algebras. Part II, Stud. Logic Found. Math., vol. 115, North-Holland Publ. Co., Amsterdam, 1985. MR0781930 (86m:03095b)

[Hi] P. J. Higgins, Algebras with a scheme of operators, Math. Nachr. 27 (1963), 115-132. MR0163940 (29:1239)

[M] S. MacLane, Categories for the working mathematician, Grad. Texts in Math., vol. 5, SpringerVerlag, New York-Berlin, 1971. MR0354798 (50:7275)

[MR1] G. Baumslag, A. Myasnikov, and V. Remeslennikov, Algebraic geometry over groups. I, J. Algebra 219 (1999), no. 1, 16-79. MR.1707663 (2000j:14003)

[MR2] A. Myasnikov and V. Remeslennikov, Algebraic geometry over groups. II. Logical foundations, J. Algebra 234 (2000), 225-276. MR1799485 (2001i:14001)

[P1] B. Plotkin, Varieties of algebras and algebraic varieties, Israel J. Math. 96 (1996), part B, 511-522. MR1433704 (98c:08011)

[P2] - Algebras with the same (algebraic) geometry, Trudy Mat. Inst. Steklov. 242 (2003), 176-207; English transl., Proc. Steklov Inst. Math. 2003, no. 3 (242), 165-196. MR2054494 (2005d:08013)

[P3] Some notions of algebraic geometry in universal algebra, Algebra i Analiz 9 (1997), no. 4, 224-248; English transl., St. Petersburg Math. J. 9 (1998), no. 4, 859-879. MR.1604318 (98j:08003)

[P4] Seven lectures on the universal algebraic geometry, Preprint (2002), Arxiv:math, GM/0204245, 87pp. 
[P5] - Varieties of algebras and algebraic varieties. Categories of algebraic varieties, Siberian Adv. Math. 7 (1997), no. 2, 64-97. MR1481222 (99a:08004)

[P6] Algebraic geometry in first order logic, Itogi Nauki i Tekhniki Ser. Sovrem. Mat. i Prilozhen., vol. 22, VINITI, Moscow, 2004, pp. 16-62; English transl., J. Math. Sci. (N.Y.) 137 (2006), no. 5, 5049-5097; http://arxiv.org/abs/math.GM/0312485.

[P7] Some results and problems related to universal algebraic geometry, Internat. J. Algebra Comput. 17 (2007), nos. 5-6, 1133-1164. MR.2355690

[PP] B. Plotkin and T. Plotkin, An algebraic approach to knowledge base models informational equivalence, Acta Appl. Math. 89 (2005), no. 1-3, 109-134 (2006); http://arxiv.org/ abs/math. GM/0312428. MR2220200 (2006m:08011)

Einstein Institute of Mathematics, Edmond J. Safra Campus, Givat Ram, Hebrew UniverSity of Jerusalem, 91904, Jerusalem, IsRael

E-mail address: plotkin@macs.biu.ac.il, borisov@math.huji.ac.il

Department of Mathematics, Bar-Ilan University, 52900, Ramat Gan, Israel

Received 15/MAY/2007

Originally published in English 